\title{
1 The DAXX-SREBP axis promotes oncogenic lipogenesis and tumorigenesis
}

2 Iqbal Mahmud ${ }^{1,2,3}$, Guimei Tian ${ }^{1}$, Jia Wang ${ }^{1,4}$, Jessica Lewis ${ }^{1}$, Aaron Waddell ${ }^{1}$, McKenzie L.

3 Lydon ${ }^{1}$, Lisa Y. Zhao ${ }^{1,7}$, Jian-Liang Li ${ }^{5}$, Hamsa Thayele Purayil ${ }^{1}$, Zhiguang Huo ${ }^{6}$, Yehia Daaka1,

4 Timothy J. Garrett ${ }^{2,3}$, Daiqing Liao ${ }^{1^{*}}$

$6{ }^{1}$ Department of Anatomy and Cell Biology, UF Health Cancer Center, University of Florida

7 College of Medicine, 2033 Mowry Road, Gainesville, FL 32610-0235, USA

$8{ }^{2}$ Southeast Center for Integrated Metabolomics, Clinical and Translational Science Institute,

9 University of Florida, Gainesville, FL 32610-0135, USA

$10{ }^{3}$ Department of Pathology, Immunology and Laboratory Medicine, University of Florida College

11 of Medicine, 1600 Archer Road, Gainesville, FL 32610, USA

$12{ }^{4}$ Affiliated Dongzhimen Hospital, Beijing University of Chinese Medicine, Beijing, P. R. China

$13{ }^{5}$ Integrative Bioinformatics, National Institute of Environmental Health Sciences, Research

14 Triangle Park, NC 27709, USA

$15{ }^{6}$ Department of Biostatistics, University of Florida, 2004 Mowry Road, Gainesville, FL 32611, USA

$16{ }^{7}$ Present Address: Department of Medicine, University of Florida College of Medicine, 1600

17 Archer Road, Gainesville, FL 32610, USA

19 * Correspondence and material requests should be addressed to D. Liao (dliao@ufl.edu).

21 Running Title: The DAXX-SREBP axis in lipogenesis 


\section{ABSTRACT}

24 De novo lipogenesis produces lipids for membrane biosynthesis and cell signaling. Elevated

25 lipogenesis is a major metabolic feature in cancer cells. In breast and other cancer types, genes

26 involved in lipogenesis are highly upregulated, but the mechanisms that control their expression

27 remain poorly understood. DAXX modulates gene expression through binding to diverse

28 transcription factors although the functional impact of these diverse interactions remains to be

29 defined. Our recent analysis indicates that DAXX is overexpressed in diverse cancer types.

30 However, mechanisms underlying DAXX's oncogenic function remains elusive. Using global

31 integrated transcriptomic and lipidomic analyses, we show that DAXX plays a key role in lipid

32 metabolism. DAXX depletion attenuates, while its overexpression enhances, lipogenic gene

33 expression, lipid synthesis and tumor growth. Mechanistically, DAXX interacts with SREBP1 and

34 SREBP2 and activates SREBP-mediated transcription. DAXX associates with lipogenic gene

35 promoters through SREBPs. Underscoring the critical roles for the DAXX-SREBP interaction for

36 lipogenesis, SREBP2 knockdown attenuates tumor growth in cells with DAXX overexpression,

37 and a DAXX mutant unable to bind SREBPs are incapable of promoting lipogenesis and tumor

38 growth. Our results identify the DAXX-SREBP axis as an important pathway for tumorigenesis. 


\section{INTRODUCTION}

40 Cancer cells exhibit elevated de novo intracellular lipogenesis, resulting in increased levels of

41 fatty acids, membrane phospholipids, and cholesterol (1). Notably, de novo lipogenesis

42 contributes minimally to the overall lipid content of normal non-proliferating cells, which generally

43 rely on the uptake of lipids from the circulation. In contrast, highly proliferative cancer cells show

44 strong avidity to acquire elevated lipids and cholesterol through either enhancing the uptake of

45 exogenous (or dietary) lipids and lipoproteins or hyperactivating their endogenous de novo lipid

46 synthesis mechanism $(1,2)$. Increased de novo lipogenesis in cancer cells is thought to supply

47 lipids for the synthesis of membranes and signaling molecules during rapid cell proliferation and

48 tumor growth, due to limited availability of lipids from the circulation in the tumor microenvironment

49 (1,3). De novo lipogenesis is controlled by several transcription factors, such as the sterol

50 regulatory element-binding proteins, SREBP1 and SREBP2 (SREBP1/2), that have been shown

51 to play an important role in maintaining lipid synthesis in cancer (4). SREBP1/2 precursors are

52 sequestered in endoplasmic reticulum. When sterol supply is low, SREBP1/2 are transported to

53 the Golgi apparatus where they are cleaved by proteases, and the N-terminal domains of SREBPs

54 are then released and imported into the nucleus to promote transcription of genes that contain

55 the sterol regulatory elements (SRES) required for lipogenesis.

57 Independently of intracellular lipid levels, oncogenic drivers, including KRAS and PI3K, promote 58 de novo lipogenesis in $\mathrm{BC}$ and other cancer types converging on mTORC1 activation (1,5-7).

59 mTORC1 promotes S6K1-dependent SREBP1/2 processing (8). The phosphatidate phosphatase

60 Lipin-1 sequesters mature SREBP1/2 in the nuclear lamina, thereby preventing SREBP1/2 from

61 activating gene expression. mTORC1 directly phosphorylates Lipin-1, which inhibits its nuclear

62 translocation and thus restores SREBP activity (9). mTOR signaling also indirectly stabilizes

63 SREBP1/2 by opposing phosphorylation-dependent ubiquitination of SREBP1/2 by the E3

64 ubiquitin ligase FBXW7 and subsequent proteasomal degradation (10-12). Notably, tumors 
65 efficiently convert acetate to acetyl-CoA (13), which is predominantly used for lipid synthesis (14),

66 highlighting the need for cancer cells to activate lipogenic enzymes (15). While the dependence

67 on de novo lipogenesis in cancer is well documented, the mechanisms that control SREBP-

68 mediated transcription underlying oncogenic de novo lipogenesis remain poorly understood.

70 DAXX, originally discovered as a context-dependent regulator of cell death or survival (16-18),

71 has an extensively documented role in transcription regulation through interacting with

72 transcription factors including p53 (19) and NF-кB (20). More recent studies have defined DAXX

73 as a specific chaperone for the histone variant H3.3 (21-23). DAXX binds specifically to the

$74 \mathrm{H} 3.3 / \mathrm{H} 4$ dimer and deposits it onto chromatin $(24,25)$. Emerging evidence suggests that DAXX

75 has an oncogenic role in diverse cancer types $(26,27)$, which appears to be linked to its functions

76 in gene regulation $(18,27,28)$. Whereas the levels of DAXX expression directly correlate with its

77 ability to promote tumor growth $(18,26-28)$, the molecular mechanisms underlying DAXX's

78 oncogenic function remain to be defined.

80 In this study, we identified DAXX as a novel regulator of oncogenic lipogenesis through its

81 interaction with SREBP1/2, leading to activating lipogenic gene expression programs and the

82 promotion of cancer cell proliferation in vitro and tumor growth in vivo. Our studies define the

83 DAXX-SREBP axis as a previously unrecognized oncogenic pathway. 
MATERIALS AND METHODS

Cell culture

87 Cell lines used for this study were obtained from ATCC (Manassas, VA) and authenticated by

88 Genetica DNA Laboratories (Burlington, NC). Cells were cultured in Dulbecco's Modified Eagle's

89 Medium (DMEM with $4.5 \mathrm{~g} / \mathrm{L}$ glucose, L-glutamine and sodium pyruvate, Corning, Tewksbury,

$90 \mathrm{MA}$ ) with $10 \%$ bovine calf serum (HyClone, GE Healthcare Bio-Sciences, Pittsburgh, PA),

91 penicillin (10 units $/ \mathrm{mL})$, and streptomycin $(10 \mu \mathrm{g} / \mathrm{mL})$ (the complete DMEM medium). The T47D

92 cell line was cultured in DMEM plus 10\% fetal bovine serum (Atlanta Biologics, Atlanta, GA),

93 penicillin (10 units $/ \mathrm{mL})$, and streptomycin $(10 \mu \mathrm{g} / \mathrm{mL})$. To culture cells in serum starvation

94 condition, serum-containing medium was removed from cell cultures after overnight culture and

95 the culture was washed once with phosphate-buffered saline (PBS, without calcium and

96 magnesium, Corning). Cells were then cultured in serum-free DMEM. For culturing cells in

97 suspension (3D culture), plates were coated with a 1:1 mixture of Matrigel (Corning) and complete

98 DMEM medium. A desirable number of cells were suspended in the Matrigel and medium mixture

99 and layered on the top of the solidified Matrigel. Complete DMEM medium was added after the

100 Matrigel was solidified. Medium was replaced with fresh complete medium every three days.

101 Colonies were imaged under a microscope; colony numbers and sizes were quantified.

\section{DNA constructs}

104 cDNAs for wild-type (WT) DAXX and mutants with a 5' coding sequence for the FLAG epitope tag 105 and a 3' coding sequences for the MYC and 6x His tags were cloned into a lentiviral vector under

106 the control of the cytomegalovirus immediate early (CMV IE) promoter. GFP-DAXX constructs

107 were cloned in the pEGFP-C2 vector. A short hairpin RNA (shRNA) targeting the DAXX coding

108 sequence (nucleotide 624-642, 5'-GGAGTTGGATCTCTCAGAA-3') was cloned into a lentiviral

109 vector under the control of the human $\mathrm{H} 1$ promoter. An shRNA construct with a scrambled 
110 sequence (Plasmid \# 36311) was from Addgene. Expression vectors for mature SREBP1a

111 (Plasmid \# 26801), mature SREBP1c (Plasmid \# 26802), and mature SREBP2 (Plasmid \# 26807)

112 were purchased from Addgene. The shRNA clones for SREBF1 (TRCN0000020607 and

113 TRCN0000020605), and SREBF2 (TRCN0000020667 and TRCN0000020668) were from the

114 human pLKO.1 TRC Library collection at the University of Florida. The SREBF2 shRNA vector

115 TRCN0000020667 was used to knockdown SREBF2 expression in MDA-MB-231 cells with DAXX

116 OE. A SREBF2 promoter fragment was PCR amplified from the genomic DNA isolated from MDA-

117 MB-231 cell line and cloned at sites upstream of the firefly luciferase reporter by the Gibson

118 assembly method. The DNA sequence was confirmed by Sanger sequencing. The PCR primers

119 are shown in Supplementary Table S1. Stable expression of cDNA and shRNA was established

120 through lentiviral transduction of cell lines and puromycin $(2 \mu \mathrm{g} / \mathrm{mL})$ selection. The derived cell

121 lines were cultured with DMEM without puromycin.

\section{Microarray, RNA-seq and qRT-PCR}

124 Cells were cultured in the complete DMEM or serum-free DMEM, and total RNAs were isolated

125 using the RNeasy kit (Qiagen) for microarray and RNA-seq analysis. For microarray experiments,

126 the RNAs were then processed for microarray hybridization to the Affymetrix GeneChip Human

127 Transcriptome Array 2.0 as described previously $(29,30)$. Total RNAs were used for RNA-seq

128 library constructions and sequencing was done with $20 \mathrm{M}$ raw reads/sample using the Illumina

129 Platform PE150 at Novogene Corporation Inc. (Sacramento, CA).

131 For quantitative real-time PCR (RT-qPCR), the isolated RNAs were reverse transcribed with

132 random hexamers using $2 \mu \mathrm{g}$ of total RNA, an RNase inhibitor, and reagents in the Multiscribe

133 reverse transcriptase kit (Life Technologies). The resulting cDNAs were diluted and used as input

134 for qPCR using the SYBR green detection method. The relative levels of gene expression were 
135 determined using the $\Delta \Delta \mathrm{Ct}$ method with the $\mathrm{Ct}$ values of $\mathrm{ACTB}$ expression as the common

136 normalizer. The primers for qPCR and other applications are shown in Supplementary Table S1.

138 Immunoprecipitation (IP) and Immunoblotting

139 Cell pellets were resuspended in the IP lysis buffer (50 mM Tris- $\mathrm{HCl}, \mathrm{pH}$ 7.5, 0.5\% Igepal-CA630, $1405 \%$ glycerol, $150 \mathrm{mM} \mathrm{NaCl}, 1.5 \mathrm{mM} \mathrm{MgCl}_{2}$, and $25 \mathrm{mM} \mathrm{NaF}$ ) containing 100 -fold diluted protease

141 inhibitor cocktail (Millipore-Sigma P8340). The cell suspension was subjected to two

142 freezing/thawing cycles. The cell lysates were then centrifuged at $15,000 \mathrm{rpm}$ at $4^{\circ} \mathrm{C}$ for $20 \mathrm{~min}$.

143 The supernatant was used for IP with a control or an antibody to a specific protein at $2 \mu \mathrm{g}$ per IP

144 in the presence of protein A-agarose beads. The beads were resuspended in the IP lysis buffer

145 along with one fifth of the volume of the $6 x$ SDS sample buffer $(0.375 \mathrm{M}$ Tris $-\mathrm{HCl}, \mathrm{pH} 6.8,12 \%$

146 SDS, $60 \%$ glycerol, $0.6 \mathrm{M} \mathrm{DTT}$, and $0.06 \%$ bromophenol blue). Samples were heated at $95^{\circ} \mathrm{C}$ for

$1475 \mathrm{~min}$ and chilled on ice for $2 \mathrm{~min}$. After brief centrifugation, the samples were loaded on a 4-20\%

148 gradient gel (Novex Tris-Glycine Mini Gels, ThermoFisher). Proteins were then electrotransferred

149 to an Immobilon®-P polyvinylidene fluoride (PVDF) membrane (Millipore). Membrane was

150 blocked with $5 \%$ non-fat milk, incubated with a primary antibody and a proper secondary antibody.

151 The proteins were detected using a chemiluminescent detection kit (Millipore) and the Fuji Super

152 RX-N X-ray films or an Amersham Imager 680.

154 For immunoblotting analyses of cell lysates of monolayer cultures, medium was removed from 155 culture plates and $1 \times$ Passive Lysis buffer (Promega) was added. The plates were frozen at $-80^{\circ} \mathrm{C}$ 156 overnight and then thawed at room temperature. The lysates were transferred to a centrifuge tube.

157 To prepare tumor lysates, xenograft tumor tissues were fragmented in the presence of liquid 158 nitrogen, approximately $50 \mathrm{mg}$ of tumor fragment was homogenized in $1 \mathrm{~mL}$ of $1 \mathrm{X}$ RIPA lysis 159 buffer on ice using a micro-homogenizer. After brief sonication at a low power output for 5 sec on 160 ice, the lysates were cleared by centrifugation at $13,000 \mathrm{rpm}$ for $15 \mathrm{~min}$ at $4{ }^{\circ} \mathrm{C}$. Protein contents 
161 were quantified using a Qubit protein assay kit. Protein extracts from cell culture or tumor lysates

162 were subjected to SDS-PAGE and electro-blotting as above. The antibodies used for this study

163 are listed in Supplementary Table S2.

164

165 Proximity Ligation Assay (PLA)

166 The PLA reagents were obtained from Millipore-Sigma (DUO92101-1KT). The assays were

167 performed following the manufacturer's protocol. The antibodies against SREBP2 (Abcam, 168 ab30682), SREBP1 (ProteinTech, 4088-1-AP), DAXX (5G11 hybridoma supernatant) were used

169 for the PLA experiments. The number of PLA signal dots was quantified as described previously $170 \quad(31)$.

\section{De novo lipogenesis assays}

173 Cells (0.5 million per well) were plated in a 6-well plate in complete DMEM medium in triplicate.

174 At 24h after seeding, cells were washed once with PBS and cultured in serum-free DMEM for 16

$175 \mathrm{~h} ; 5 \mu \mathrm{Ci}$ of $\left[1{ }^{-14} \mathrm{C}\right]$ acetate (NEC084H001MC, Perkin Elmer, Waltham, MA, USA) per mL was 176 added and the cells were cultured for four more hours. Cells were then washed twice with PBS

177 and trypsinized. Cells were pelleted and resuspended in $0.5 \mathrm{~mL}$ of $0.5 \%$ Triton $\mathrm{X}-100$. The protein 178 concentration of the lysates was determined for normalization. The lysates were extracted with 179 ice cold chloroform/methanol $(2: 1 \mathrm{v} / \mathrm{v})$. After centrifugation at 1,000 rpm for $20 \mathrm{~min}$, the organic 180 phase was collected and air dried. The radioactivity was determined with a liquid scintillation 181 counter (Beckman LS 5000TD). The radioactivity was normalized against protein concentration.

\section{Liquid chromatography (LC)-mass spectrometry (MS) experiments}

184 For lipid analysis, we used these internal lipid standards: triglyceride (TG 15:0/15:0/15:0 and TG 185 17:0/17:0/17:0, Sigma-Aldrich), lysophosphatidylcholines (LPC, 17:0 and 19:0), 186 phosphatidylcholines (PC, 17:0/17:0 and 19:0/19:0), phosphatidylethanolamines (PE, 15:0/15:0 
187 and 17:0/17:0), phosphatidylserines (PS, 14:0/14:0 and 17:0/17:0), and phosphatidylglycerols

188 (PG, 14:0/14:0 and 17:0/17:0) (Avanti Polar Lipids, Alabaster, AL). The lipid standards were 189 dissolved in 2:1 (v/v) chloroform/methanol to make a 1000 ppm stock solution and a working 100 190 ppm standard mix was then prepared by diluting the stock solution with the same solvent mixture.

191 For sample normalization, total protein concentration in each sample was determined using a

192 Qubit 3.0 Fluorometer.

193

194 Cell lines with a control vector, an shRNA against an indicated gene, WT DAXX, or the del 327-

195335 mutant were cultured with the complete DMEM. When cells grew to approximately $80 \%$ 196 confluency, they were washed twice with PBS and cells were detached using a cell lifter. Cell 197 pellets were washed twice with $40 \mathrm{mM}$ ammonium formate (AF). The cell pellets were 198 resuspended in $50 \mu \mathrm{L}$ of $\mathrm{AF}$ with vortex in a glass vial and subjected to high efficient bead beater 199 cell disruption to release intracellular lipids. A small amount of the homogenized cell pellet was 200 taken for Qubit protein concentration determination. Lipids were extracted by adding ice-cold 201 chloroform $(2 \mathrm{~mL})$ and methanol $(1 \mathrm{~mL})$ along with $20 \mu \mathrm{L}$ of internal standard mixtures. The 202 extraction mixture was incubated on ice for $1 \mathrm{~h}$ with occasional vortex mixing. Finally, $1 \mathrm{~mL} \mathrm{H}_{2} \mathrm{O}$ 203 was added to the mixture, which was incubated for 10 min with occasional vortex mixing. Samples 204 were then centrifuged at 2,000 rpm for $5 \mathrm{~min}$. The lower phase (organic layer) was collected in a 205 separate glass vial and subjected to dry under nitrogen gas at $30^{\circ} \mathrm{C}$ using a dryer (MultiVap, 206 Organomation Associates). Dried samples were reconstituted by adding $50 \mu \mathrm{L}$ isopropyl alcohol 207 and transferred to a glass LC vial with insert. Samples were loaded to an auto-sampler at $5^{\circ} \mathrm{C}$.

209 For analyzing lipids, we ran samples for quality control (QC) in each instrument run. A pooled QC 210 sample (a $25 \mu \mathrm{L}$ aliquot) for each extraction was injected after analyzing every five samples. The 211 pooled QC sample was run to assess system reproducibility, and a blank (solvent mixture only) 212 was used to flush the column. We did not observe any changes regarding the number of 
213 background ions, which always corresponded to the specific solvent used for lipid extraction. Also,

214 we did not notice any effects on reproducibility of ion source regardless of solvents used for

215 extraction. The stability and repeatability of the instruments were evaluated using identical neat

216 QC samples (a mixture of all internal standards in deuterated form) throughout the process of

217 sample injection. Principal component analysis (PCA) was performed to evaluate the variation of

218 QC samples. All neat QC samples clustered together, confirming the stability and reproducibility

219 of our experimental lipid analysis system.

221 For data collection, processing, and analysis, we used a Dionex Ultimate 3000 UHPLC system

222 coupled to a $\mathrm{Q}$ Exactive ${ }^{\mathrm{TM}}$ hybrid quadrupole-orbitrap mass spectrometer operated in $\mathrm{HESI}$ -

223 positive and negative ion mode. A Supelco Analytical Titan reverse-phase column (RPC) C18

$224\left(2.1 \times 75 \mathrm{~mm}\right.$ with $1.9 \mu \mathrm{m}$ monodisperse silica) equilibrated at $30^{\circ} \mathrm{C}$ with solvents $\mathrm{A}$ (acetonitrile

225 and water 60:40, v/v) and B (isopropyl alcohol, acetonitrile, and water 90:8:2, v/v/v) as mobile

226 phases was used for data collection. The flow rate was $0.5 \mathrm{~mL} / \mathrm{min}$, and the injection volume was

$2275 \mu \mathrm{L}$. The total run time was $22 \mathrm{~min}$, including a 2-min equilibration. The MS conditions for positive

228 and negative ion modes were spray voltage at $3.5 \mathrm{kV}$, sheath gas at 30 arbitrary units, sweep gas

229 at 1 arbitrary unit, auxiliary nitrogen pressure at 5 arbitrary units, capillary temperature at $300{ }^{\circ} \mathrm{C}$,

230 HESI auxiliary gas heater temperature at $350^{\circ} \mathrm{C}$, and S-lens RF at 35 arbitrary units. The

231 instrument was set to acquire in the mass range of most expected cellular lipids and therefore

$232 \mathrm{~m} / \mathrm{z}$ 100-1500 was chosen with a mass resolution of 70,000 (defined at $\mathrm{m} / \mathrm{z} 200$ ). Global lipid

233 profiling was performed using full scan and ddMS2 (data dependent MS-MS).

235 Data were recorded from 0.0 to $17 \mathrm{~min}$ as total ion chromatography (TIC) and then corresponding

236 MS data were extracted using Thermo Xcalibur (version 2.2.44). After data collection, raw data

237 files were converted to mzXML format using the Proteowizard MSConvert software. MZmine 2.15

238 (freeware) was used for mass detection with mass detector centroid noise set at 1.0E05 using 
only MS level 1 data; chromatogram building and deconvolution were then applied $(\mathrm{m} / \mathrm{z}$ tolerance, 0.005 or $10 \mathrm{ppm}$; retention time tolerance, $0.2 \mathrm{~min}$; minimum time span, $0.1 \mathrm{~min}$; and minimum

241 height, 5.0E05) followed by isotope grouping, alignment (m/z tolerance, 0.005 or 10 ppm;

242 retention time tolerance, $0.2 \mathrm{~min})$, and gap filling ( $\mathrm{m} / \mathrm{z}$ tolerance, 0.005 or $10 \mathrm{ppm}$; retention time

243 tolerance, $0.2 \mathrm{~min}$, and intensity tolerance $25 \%$ ). MZmine-based online metabolite search engine

244 KEGG, MMCD database, XCMS online database, Metaboanalyst 3.0, R program, and internal

245 retention time library were used for the identification and analysis of metabolites.

\section{In vivo tumor growth}

248 All mice were maintained under pathogen-free conditions. Female NSG (NOD.Cg249 Prkdc $\left.{ }^{\text {scid }} / \mid 2 \mathrm{rg}^{\mathrm{tm} 1 \mathrm{~W}_{\mathrm{j}} \mathrm{l}} / \mathrm{SzJ}\right)$ mice, between the ages of 4-6 weeks, were injected subcutaneously in a 250 mammary fat-pad area with one million cells in $100 \mu \mathrm{L}$ of complete DMEM (MDA-MB-231-derived 251 cell lines) or in a suspension of $50 \mu \mathrm{L}$ of Matrigel and $50 \mu \mathrm{L}$ of cell suspension (MDA-MB-468252 derived cell lines). Tumor growth was monitored by measuring tumor dimensions using a digital 253 caliper once a week until endpoint. Tumor volume was calculated with the formula $1 / 2 \mathrm{x}$ length $\mathrm{x}$ 254 width ${ }^{2}$. At the endpoint, mice were euthanized, tumors were excised, weighted, and photographed. 255 Tumor lysates were prepared for immunoblotting analysis. Animal use has been approved for this 256 project by the University of Florida IACUC.

\section{Chromatin immunoprecipitation (ChIP)}

259 The panel of MDA-MB-231-derived cell lines (control and WT DAXX OE) were cultured in 260 complete DMEM. ChIP experiments were performed essentially as described (32). Briefly, at 261 about $90 \%$ confluency, the cells were crosslinked by adding $37 \%$ formaldehyde to the final 262 concentration of $1 \%$ for 10 min at room temperature. Crosslinking was stopped by adding glycine 263 to the final concentration of $125 \mathrm{mM}$. Cells were lifted, washed with cold PBS, and pelleted by 264 centrifugation. The cells were resuspended in a swelling buffer in the presence of the protease 
265 inhibitor cocktail (Sigma) and then pelleted and resuspended in the SDS lysis buffer. The lysates

266 were transferred to a Covaris microTUBE and sonicated with an E220 Covaris Ultrasonicator.

267 Chromatin fragmentation ( $500 \mathrm{bps})$ was verified by agarose gel electrophoresis. The fragmented

268 chromatins were diluted and incubated with a control IgG and the DAXX mAb (5G11) along with

269 protein A/G magnetic beads. The beads were washed sequentially with a low salt buffer, high salt

270 buffer, LiCl buffer, and TE buffer (twice). The immunoprecipitated chromatins were eluted at $65^{\circ} \mathrm{C}$

271 for $15 \mathrm{~min}$, and the eluted chromatins were subjected to proteinase $\mathrm{K}$ digestion at $65^{\circ} \mathrm{C}$ for $3 \mathrm{~h}$.

272 The DNAs were recovered through a Qiagen mini-prep column. The immunoprecipitated DNAs

273 were used for qPCR and library construction and high throughput sequencing using an Illumina

274 Hi-Seq 2500 sequencer.

275

\section{Bioinformatics analysis}

277 We analyzed gene expression based on publicly available datasets. Gene expression data for 278 normal, benign, primary, and metastatic tumor samples were included for our analysis.

279 Normalized expression levels for specific genes were compared between different sample types.

280 Computations were conducted in R statistical package (https:// www.r-project.org/) and in

281 GraphPad Prism 7.0. For Ingenuity Pathway Analysis (IPA), genes that were differentially 282 expressed (fold-change over \pm 1.3 and $p$-value $<0.05$ ) were used for the Ingenuity Pathway 283 Analysis (Ingenuity Systems, Qiagen Bioinformatics, http://www.ingenuity.com). Gene Set 284 Enrichment Analysis (GSEA) was performed using the Java desktop software 285 (http://software.broadinstitute.org/gsea/index.jsp), as described previously (33). The GSEA tool 286 was used in pre-ranked mode with all default parameters. For microarray data analysis, probe set 287 files (.cel file) were normalized by RMA algorithm and analyzed using both R statistical package 288 as well as Affymetrix expression and transcriptome console software from ThermoFisher 289 Scientific. For RNA-seq data analysis, we used the RNAseq data analysis pipeline reported 290 previously (34). Briefly, fastq files were aligned to Genome Reference Consortium Human Build 
29138 (GRCh38) using HISAT2 (35); the transcripts assembling was performed using StringTie (36)

292 with RefSeq as transcripts ID; and the normalized counts (by FPKM) was called using Ballgown

293 (37). The differential expression analysis was performed using R package limma (38); and the

294 pathway enrichment analysis was performed using ingenuity pathway analysis. ChIP-seq

295 sequencing reads (Fastq files) were mapped to the human genome (GRCh37/hg19) using

296 Bowtie2 (39), where option -local was specified to trim or clip unaligned reads from one or both

297 ends of the alignment. Genome browser BedGraph tracks and read density histograms were

298 generated using SeqMINER. Peak finding and annotation to the nearest Refseq gene promoter

299 was performed and de novo motif discovery was carried out using HOMER (40).

\section{Statistical analysis}

302 Gene expression assays were conducted in two to three biological replicates. Metabolic profiling 303 assays were performed in four to six replicates. Data are presented as the mean along with 304 standard error of the mean (SEM). Student's t-test was used to compare two groups of 305 independent samples. For all data analysis, $\mathrm{p}<0.05$ was considered statistically significant. 


\section{RESULTS}

\section{Transcriptomic profiling implicates DAXX in promoting lipogenic gene expression}

309 Bioinformatic analyses of clinical BC samples of The Cancer Genome Atlas (TCGA) and the

310 Clinical Proteomic Tumor Analysis Consortium (CPTAC) datasets revealed that DAXX mRNA and

311 protein levels are elevated in all four major BC subtypes with highest levels in the triple-negative

$312 B C(T N B C)$ subtype (Figure $1 A$ and $B$ ), suggesting a potential oncogenic role for DAXX (18). To

313 understand the function of DAXX in cancer, we used gain and loss of function approaches:

314 genetically depleted endogenous DAXX or overexpressed wild-type (WT) DAXX in the TNBC cell

315 line MDA-MB-231 (Figure 1C). Transcriptomic analyses using microarray and RNA-seq revealed

316 distinct gene expression profiles for cells with DAXX mRNA knockdown (KD) and WT DAXX

317 overexpression (OE) in comparison to control (CTL) cells (Figure 1D and E). Unexpectedly,

318 Ingenuity Pathway Analysis (IPA) of differentially expressed genes in cells with DAXX KD or OE

319 in comparison to CTL cells revealed a marked downregulation and upregulation, respectively, of

320 the de novo lipogenesis pathways. Lipogenesis regulators (SREBF1/2 encoding SREBP1/2 and

321 SCAP) were among the most highly inhibited upstream regulators in the KD cells, while WT DAXX

322 OE activated SREBF1/2 (Figure 1F). Correspondingly, the cholesterol biosynthesis via the

323 mevalonate pathway were among the top canonical pathways identified by IPA (Supplementary

324 Figure S1A). Most of the genes in the biosynthesis of cholesterol, fatty acids, glycerolipid, and

325 glycerophospholipids are affected by DAXX expression levels (Figure 1E). Gene Set Enrichment

326 Analysis (GSEA) of transcriptomic data confirmed suppression and activation of the de novo

327 lipogenesis pathway by DAXX KD and WT OE, respectively (Supplementary Figure S1B and C).

328 Of note, several transcriptional regulators that are known to interact with DAXX such as JUN and

329 PML (18) were also affected by DAXX expression levels. Interestingly, the insulin receptor (INSR)

330 pathway that regulates intracellular lipid production (41) also seems to be positively regulated by

\section{DAXX (Figure 1F).}


333 RT-qPCR analyses provided validation for the microarray results (Supplementary Figure S2). The

334 impact of DAXX knockdown or overexpression on lipogenic gene expression was further validated

335 by immunofluorescence microscopy and immunoblotting (Supplementary Figure S2B and C).

336 Using a tetracycline-inducible gene expression system, we found that DAXX induction increased

337 lipogenic gene expression (Supplementary Figure S2D), providing further evidence that DAXX

338 directly activates lipogenic gene expression. In keeping with our findings, our analysis of public

339 gene expression datasets based on human and mouse cells (42-44) indicated that DAXX is

340 involved in promoting the SREBP/lipogenesis pathway (Supplementary Figure S3). Further

341 analyses of the TCGA data indicate that DAXX expression levels positively correlate with that of

342 SREBP1 and SREBP2 (Supplementary Figure S5) and a panel of lipogenic genes (Figure 1G).

343 Notably, high mRNA levels of the gene set including DAXX shown in Figure 1G predict poor

344 patient survival (Figure $1 \mathrm{H}$ ). Collectively, these data provide evidence that DAXX may play an

345 important role in promoting lipogenic gene expression.

\section{DAXX promotes lipid production}

348 To determine whether transcriptomic differences correspond to an alteration of intracellular

349 lipidome, the same panel of MDA-MB-231-derived cell lines used for our transcriptomic study

350 were subjected to global lipidome analysis using LC-MS technique. Similar to transcriptomic

351 profiles, control, DAXX KD and OE cells cluster into groups with distinct intracellular lipidomes

352 (Figure 2A and B). A lipidome-based pathway analysis again revealed that DAXX expression

353 levels significantly impact lipogenesis pathways (Figure 2C). To validate DAXX's role in

354 lipogenesis, we depleted DAXX using CRISPR/Cas9 (Supplementary Figure S5A). Lipidomic

355 profiling again showed that DAXX depletion significantly altered lipidomes (Supplementary Figure 356 S5B-E). 
358 To understand the broader role of DAXX in lipogenesis, we have explored correlation of DAXX

359 expression with lipid production in different cancer types. We have depleted endogenous DAXX

360 or overexpressed wild-type DAXX in another TNBC cell line MDA-MB-468 (Figure 2D).

361 Consistent with lipidomic changes in cells derived from MDA-MB-231, global lipidomic profiling

362 revealed that DAXX KD reduced, but WT OE increased levels of diverse lipid molecules,

363 respectively, in MDA-MB-468-derived cells (Figure 2E-G). Principal component analysis (PCA)

364 analysis indicated that MDA-MB-468 cells with different levels of DAXX expression exhibit distinct

365 lipid profiles (Figure 2E). Pathway analyses based on metabolites identified the biosynthesis

366 pathways of glycerophospholipid, steroid, glycerolipid and fatty acid metabolism as the top

367 pathways influenced by DAXX expression levels (Figure 2G).

It is well known that cancer cells utilize acetate as an alternative carbon source to glucose for de novo lipogenesis (6). To assess whether DAXX affects acetate-driven de novo lipogenesis, we

371 treated cells that express different levels of DAXX with $\left[{ }^{14} \mathrm{C}\right]$-acetate. Quantification of the $\left[{ }^{14} \mathrm{C}\right]-$

372 labeled lipids showed that DAXX expression levels positively correlated with levels of intracellular

373 lipids, with reduced or increased labeled lipids, respectively, in DAXX KD or DAXX OE in cells 374 derived from MDA-MB-231 and MDA-MB-468 cells (Figure 2H). Diminished de novo lipogenesis 375 upon DAXX depletion was also observed in BC cell lines of luminal subtypes (MCF7 and T47D) 376 and the colon cancer cell line HCT116 (Figure 2H). Collectively, these metabolic experiments 377 established a functional role for DAXX in de novo lipogenesis in cancer cells.

379 DAXX is critical for tumor growth and lipogenesis in vivo

380 As de novo lipogenesis is critical to cell proliferation and tumorigenesis $(1,45)$, DAXX expression 381 levels could impact cell growth in vitro and tumor growth in vivo due to alteration in lipid production.

382 Indeed, DAXX knockdown reduced the number and size of colonies when compared to control, 383 while WT DAXX OE had the opposite effects in three-dimensional cell culture model of MDA-MB- 
231 (Supplementary Figure. S6).

Next, we examined effects of DAXX expression levels on tumor growth in vivo. In orthotopic BC xenograft models using female mice, DAXX knockdown markedly reduced while WT DAXX OE significantly increased tumor growth of both MDA-MB-231 and MDA-MB-468 TNBC cell lines (Figure $3 \mathrm{~A}$ and $\mathrm{B}$ ). Despite the notable difference in the tumor growth rate between the MDAMB-231 and the MDA-MB-468 xenograft tumors, the effects of DAXX expression levels on tumor growth were clearly observed in both TNBC tumor models (Figure 3 A and B). Immunoblotting analysis of tumor extracts showed that DAXX KD and OE were maintained in in vivo (Figure 3C).

393 We profiled the lipids in xenograft tumors derived from cells with different levels of DAXX expression and lipid production. We found that the expression levels of DAXX positively correlated with elevated levels of lipids in xenograft tumors (Figure 3D). PCA analysis indicated that tumors with different DAXX expression levels exhibited distinct lipid profiles (Figure 3E). Overexpression of DAXX significantly elevated glycerophospholipids (Figure 3D), total TGs $(n=350)$, and metabolites involved in cholesterol biosynthesis (Figure 3F). Altogether, our data demonstrate that DAXX promotes oncogenic lipogenesis and tumor growth in vivo.

\section{DAXX interacts with SREBP1 and SREBP2}

402 SREBP1/2 are master transcription factors that promote lipid production when the intracellular 403 levels of lipids/sterols are low $(3,4,46)$. Because DAXX expression levels positively correlate with 404 the activation of SREBP/lipid biosynthesis pathway (Figures 1-3), we reasoned that DAXX could 405 regulate lipid biosynthesis through interacting with SREBPs. Immunoprecipitation (IP) of total cell 406 extracts with an anti-DAXX antibody evidenced co-precipitation of the precursor and mature (M) 407 forms of SREBP2 (Figure 4A). Using Proximity Ligation Assay (PLA) with a mouse monoclonal 408 anti-DAXX and a rabbit polyclonal anti-SREBP2 antibody, endogenous DAXX-SREBP2 409 interaction signals were detected in both the cytoplasm and the nucleus of the MDA-MB-231 cells 
410 (Figure. 4B). Interestingly, the number of DAXX/SREBP2 PLA signals was significantly increased

411 in the absence of serum (Figure 4B). The number of PLA signal dots were shown to be

412 proportional to cellular protein levels (31). Thus, low extracellular supply of lipids appeared to

413 enhance the DAXX-SREBP2 interaction.

415 Likewise, DAXX interacted with both the precursor and mature forms of SREBP1 in MDA-MB-231

416 cells (Figure 4E and F). To assess the interaction between DAXX and mature SREBP1, we

417 conducted co-IP using nuclear and cytoplasmic fractions of the MDA-MB-231 cells. As expected,

418 the mature SREBP1 was predominantly detected in the nucleus (Figure 4F). Notably, mature

419 SREBP1 was enriched in the DAXX immunoprecipitates of both fractions (Figure 4F). The DAXX-

420 SREBP1 interaction signals were also detected in MDA-MB-231 cells using PLA (Figure 4G).

421 Similar to the DAXX-SREBP2 interaction (Figure 4B), the number of DAXX-SREBP1 PLA foci

422 was increased in the absence of serum (Figure 4G). Altogether, these data show that DAXX

423 binds to both precursor and mature SREBP1/2.

425 Using various DAXX deletion constructs in transfected 293T cells, we found that the mature 426 SREBP2 interacted with two separate regions of DAXX, the $\mathrm{N}$-terminal part encompassing the 427 well-folded helical bundle domain termed 4HB (DAXX helical bundle) (47) and a part of the central 428 histone-binding domain (HBD) (24) (Figure 4C and D). Interestingly, although 4HB and HBD 429 individually bound robustly to SREBP2 (Figure 4C lanes 3 and 4; Figure 4D lanes 1 and 3), the 430 integrity of both binding sites in the context of the full-length DAXX or a longer construct appeared 431 critical for the DAXX-mature SREBP2 interaction. Indeed, mutations within 4HB (I127A, del 129432 132) (Figure 4C, lane 5) or HBD (del 327-335, Figure 4C lane 6; del 191-242, Figure 4C lane 7) 433 abolished the DAXX-SREBP2 interaction. Notably, the DAXX construct (aa 1-437) lacking the 434 sequence from the acidic domain to the C-terminus seemed to show higher affinity to SERBP2 435 (Figure 4C lane 8). As deletions of C-terminal regions of DAXX did not affect the DAXX-SREBP2 
436 interaction (Figure 4C and D) and C-terminal fragments spanning aa 574-740 did not bind to

437 SREBP2 (data not shown), we concluded that SREBP2 does not bind to the C-terminal domain

438 of DAXX. The mature SREBP1a bound to DAXX in a similar fashion (Figure $4 \mathrm{H}$ ). Collectively, 439 our data demonstrate that the mature SREBP1/2 specifically interact with DAXX via DAXX's 4HB 440 and HBD (Figure 4I).

442 SREBP-binding sites are enriched in DAXX-associated chromatins

443 The data presented above suggest that DAXX promotes SREBP-mediated transcription to 444 stimulate lipogenesis. To test this idea, we conducted luciferase reporter assays. As shown in 445 Figure 5A, forced expression of mature SREBP2, SREBP1a and SREBP1c increased the activity 446 of the luciferase reporter that is under the control of the SREBF2 promoter containing a canonical 447 SRE. Co-expression of DAXX further increased the luciferase activity, while DAXX alone had only 448 minimal effects (Figure 5A).

450 We surveyed genome-wide occupancy of DAXX using the ChIP-seq technology. Overexpression 451 of WT DAXX increased DAXX's chromatin association (Figure 5B). Consistent with other studies 452 (42), DAXX primarily bound to sites in introns and intergenic regions with less frequent association 453 with promoters (Figure 5C). A de novo motif analysis revealed that SREBP-binding elements were 454 significantly enriched in DAXX-associated sites (Figure 5D and Supplementary Figure S7).

456 ChIP-qPCR experiments demonstrated that WT DAXX was enriched in the promoters of FASN, 457 ACACA and SREBF2 (Figure 5E). In MDA-MB-231 cells depleted of SREBP2, DAXX's 458 recruitment to the promoters of FASN, ACACA and SREBP2 was impaired (Figure 5F), 459 demonstrating that SREBP2 is critical for DAXX to bind the promoters of lipogenic genes. 460 Likewise, DAXX recruitment to the promoters of lipogenesis genes were impaired in MDA-MB- 
461231 cells depleted of SREBP1 (Data not shown). Altogether, these data demonstrating that

462 SREBPs are critical for DAXX to bind the promoters of lipogenic genes.

\section{The DAXX-SREBP axis is important for lipogenesis and tumor growth}

465 SREBP1/2 drive lipid biosynthesis to promote tumorigenesis $(4,46)$. In MDA-MB-231 cells, 466 SREBP2 knockdown reduced de novo lipogenesis from acetate and tumor growth in vivo (Figure $4676 \mathrm{~A}$ and $\mathrm{E})$, whereas the overexpression of mature SREBP2 increased lipogenesis and tumor 468 growth (Figure 6A and E). Concordantly, lipidomic profiling shows that SREBP2 knockdown had 469 a marked impact on global lipid landscapes (Figure 6B-D). Likewise, SREBP1 knockdown (or 470 overexpression) impaired (or promoted) de novo lipogenesis and tumor growth, respectively (data 471 not shown). SREBP1 knockdown also significantly altered intracellular lipidome (Supplementary

472 Figure 8). These data indicate both SREBP1 and SREBP2 are critical mediators of lipogenesis 473 and tumorigenesis in TNBC models. Our data presented above demonstrated that the DAXX474 SREBP interactions are critical for lipogenic gene expression, de novo lipogenesis and tumor 475 growth. To further link SREBP2 to DAXX-mediated tumorigenesis, we depleted SREBP2 in MDA476 MB-231 cells with WT DAXX OE. We observed that SREBP2 knockdown in the DAXX OE cells 477 significantly attenuated the levels of lipogenic enzymes and tumor growth (Figure 6F and G), 478 suggesting that SREBP2 is a critical effector of DAXX's oncogenic function.

480 To further assess the importance of the DAXX-SREBP interaction on lipogenesis, we 481 overexpressed a DAXX mutant (del 327-335) defective of SREBP1 or SREBP2 binding (Figure $4824 \mathrm{C}$ and $\mathrm{H}$ ) in MDA-MB-231 cells. The protein levels of both WT DAXX and the del 327-335 mutant 483 were similar (Figure $7 \mathrm{~A}$ and $\mathrm{B}$ ). A de novo lipogenesis assay using $\left[{ }^{14} \mathrm{C}\right]$-acetate labeling indicated 484 that the del 327-331 mutant attenuated lipogenesis (Figure 7C). Lipidomic profiling revealed that 485 MDA-MB-231 cells expressing the del 327-335 mutant has a distinct global lipid profile from that 486 of cells expressing the WT DAXX (Figure 7D and E) and that this mutant was impaired to enhance 
487 lipid production including glycerolipids and glycerophospholipids as compared to WT DAXX

488 (Figure 7F and G). Reduced levels of specific lipid molecules such as cholesterol and fatty acid 489 derivatives were evident in MDA-MB-231 cells expressing the del 327-331 mutant compared to 490 those expressing the WT DAXX (Figure 7H). In vivo, the growth of xenograft tumors derived from 491 cells expressing the del 327-335 mutant was markedly slower than that derived from cells with

492 WT DAXX (Figure 7I). These data collectively indicate that the DAXX-SREBP interaction is critical 493 for DAXX to promote lipogenesis and tumorigenesis (Figure 7J).

\section{DISCUSSION}

496 Lipid availability for proliferating cells determines activity of intracellular lipid biosynthesis pathway.

497 In a nutrient-poor tumor microenvironment, limited supplies of lipids necessitate the activation of

498 intracellular lipid production in tumor cells for sustained tumor growth. An elaborate sterol sensing 499 machinery controls nuclear translocation of SREBP1/2, which promote expression of enzymes 500 required for de novo lipogenesis $(1,48)$. SREBP1/2 in conjunction with other transcription factors, 501 such as the E-box-binding basic helix-loop-helix (bHLH) transcription factor USF1, activate 502 expression of lipogenic enzymes and regulators (41). Other coregulators of gene expression 503 such as acetyltransferases (e.g., p300 and PCAF) as well as oncogenic signaling pathways (e.g., 504 KRAS and mTOR) also play important roles in stimulating de novo lipogenesis $(6,49)$. We 505 demonstrated here that DAXX is important for de novo lipogenesis. Mechanistically, DAXX 506 interacts with SREBP1/2 and is enriched in chromatins containing SRE motifs. Importantly, the 507 DAXX del 327-335 mutant that cannot bind SREBP1/2 were unable to promote lipogenesis and 508 tumor growth. SREBP2 downregulation prevents enhanced tumor growth by DAXX 509 overexpression. Thus, it is likely that DAXX enhances lipogenesis through interacting with 510 SREBP1/2 to promote lipogenic gene expression, lipid production and tumorigenesis.

512 Our data suggest that DAXX acts to promote SREBP-mediated transcription. It has been well 
513 documented that DAXX can activate and repress transcription, depending on co-regulators that

514 are associated with DAXX (18). Epigenetic modifiers such as HDACs and DNA and histone

515 methyltransferases are involved in DAXX-mediated transcription repression, while coactivators

516 (e.g., CBP) are involved in DAXX-mediated gene activation. The H3.3 histone chaperone function

517 of DAXX is also implicated in both transcriptional activation $(50,51)$ and repression $(52,53)$.

518 Independently of H3.3 deposition by DAXX to chromatin, the H3.3/H4 dimer metabolically

519 stabilizes DAXX protein, which indirectly enhances repression of endogenous retroviruses by a 520 complex consisting of the DAXX-H3.3/H4 sub-complex, HDAC1, KAP1, and SETDB1 (44). Our

521 data show that SREBP1/2 bind to DAXX by contacting with both $4 \mathrm{HB}$ and HBD (Figure 4). A

522 previous structural study demonstrates that a peptide within the transactivation domain of p53

523 binds to DAXX 4HB (47). It will be interesting to assess whether DAXX also engages the

524 transactivation domain of SREBPs to promote transcription and whether the H3.3 chaperone

525 function of DAXX is important for lipogenic gene expression.

527 Of note, the binding motifs of other known DAXX-binding transcription factors such as NF- $\mathrm{B}(20)$

528 were highly enriched in DAXX ChIP-seq peaks (Figure 5D). Our ChIP-seq data also implicate 529 the chromatin recruitment of DAXX by other transcription factors such as RUNX1, RUNX2, HIC1 530 and c-MYC that were not previously shown to interact with DAXX. Furthermore, DAXX might 531 interact with the core-transcriptional machinery, as the TATA-box and DCE (downstream core 532 element) were enriched in DAXX-binding chromatins (Figure 5D and Supplementary Figure S7). 533 These observations suggest a broader role for DAXX in transcription regulation.

535 Based on our co-immunoprecipitation and PLA results, DAXX appears to interact with SREBP1/2 536 in both the cytoplasm and the nucleus (Figure 4). Interestingly, the number of DAXX-SREBP1/2 537 PLA complexes increases upon serum starvation (Figure 4), suggesting that a low level of lipid 
538 supply might trigger the formation of the DAXX-SREBP1/2 complexes in the cytoplasm. In the

539 nucleus, the DAXX-SREBP interactions are expected to mediate DAXX's chromatin recruitment

540 and the activation of lipogenic gene expression. The functional effects of DAXX-SREBP

541 interactions in the cytoplasm are currently unknown. In the cytoplasm, DAXX has been shown to

542 interact with regulators of cell death and cell survival (18). A recent study demonstrates that

543 DAXX promotes the formation of SQSTM1/p62 membrane-less liquid compartments to activate

544 cellular anti-oxidative stress response (54). The functional ramification of the interaction between

545 DAXX and SREBPs in the cytoplasm requires further investigation.

547 Targeting the de novo lipogenesis pathways such as the biosynthesis of fatty acids (1) and 548 cholesterol $(55,56)$ is a promising approach for treating BC and other cancer types. Our data 549 show that the DAXX-SREBP axis appears to play a critical role in promoting lipid production and

550 tumor growth. The specific binding interactions between DAXX and SREBP1/2 could be potential

551 therapeutic targets for anticancer drug development. It will be interesting to define precisely the 552 interfaces between DAXX and SREBPs and to determine whether these interfaces are tractable 553 as therapeutic targets. 


\section{ACKNOWLEDGEMENTS}

557 We thank Yue Li for help with initial microarray data analysis, Maria Zajac-Kaye, Shuang Huang

558 and Scott Dehm for providing reagents. We also thank Subramaniam Shyamalagovindarajan and

559 Ranjan Perera for library construction and high throughput sequencing for the ChIP-seq

560 experiments.

Author contributions: I.M. designed, and performed experiments, analyzed and interpreted data,

563 and contributed to writing; G.T., J.W., J.L., A.W., M.L.L., L.Y.Z., and H.T.P. conducted

564 experiments, acquired and analyzed data; J.J.L. performed IPA analysis; T.G. supervised mass

565 spectrometry experiments; Z.H. conducted bioinformatics analysis. Y.D. analyzed and interpreted

566 data and contributed to writing; D.L. designed and conducted experiments, analyzed and

567 interpreted data, supervised the entire study, and wrote the paper.

\section{FUNDING}

570 This work was supported by grants from Bankhead-Coley Cancer Research Program (4BF02 and 571 6BC03), and James and Esther King Biomedical Research Program (6JK03 and 20K07), Florida 572 Department of Health, Florida Breast Cancer Foundation, and UF Health Cancer Center (to D. 573 Liao). Mass spectrometry-based global lipidomics work was supported by grant from National 574 Institutes of Health (U24DK097209 to T.G.). J. Li was supported by the Intramural Research

575 Program of the $\mathrm{NIH}$, National Institute of Environmental Health Sciences. The high throughput 576 sequencing of the ChIP-seq experiments was supported by a grant from Bankhead-Coley Cancer 577 Research Program, Florida Department of Health (5BC08 to Ranjan Perara).

579 Competing interests: A US patent application related to this study has been filed on behalf of 580 the University of Florida Research Foundation. 


\section{REFERENCES}

583

584

585

586

587

588

589

590

591

592

593

594

595

596

597

598

599

600

601

602

603

604

605

606

607

608

609

610

611

612

613

614

615

616

617

618

619

620

621

622

623

1. Rohrig, F. and Schulze, A. (2016) The multifaceted roles of fatty acid synthesis in cancer. Nat Rev Cancer, 16, 732-749.

2. Beloribi-Djefaflia, S., Vasseur, S. and Guillaumond, F. (2016) Lipid metabolic reprogramming in cancer cells. Oncogenesis, 5, e189.

3. Lewis, C.A., Brault, C., Peck, B., Bensaad, K., Griffiths, B., Mitter, R., Chakravarty, P., East, P., Dankworth, B., Alibhai, D. et al. (2015) SREBP maintains lipid biosynthesis and viability of cancer cells under lipid- and oxygen-deprived conditions and defines a gene signature associated with poor survival in glioblastoma multiforme. Oncogene, 34, 51285140.

4. Griffiths, B., Lewis, C.A., Bensaad, K., Ros, S., Zhang, Q., Ferber, E.C., Konisti, S., Peck, B., Miess, H., East, P. et al. (2013) Sterol regulatory element binding protein-dependent regulation of lipid synthesis supports cell survival and tumor growth. Cancer Metab, 1, 3.

5. Guo, D., Prins, R.M., Dang, J., Kuga, D., Iwanami, A., Soto, H., Lin, K.Y., Huang, T.T., Akhavan, D., Hock, M.B. et al. (2009) EGFR signaling through an Akt-SREBP-1-dependent, rapamycin-resistant pathway sensitizes glioblastomas to antilipogenic therapy. Sci Signal, 2, ra82.

6. Ricoult, S.J., Yecies, J.L., Ben-Sahra, I. and Manning, B.D. (2016) Oncogenic PI3K and KRas stimulate de novo lipid synthesis through mTORC1 and SREBP. Oncogene, 35, 12501260.

7. Saxton, R.A. and Sabatini, D.M. (2017) mTOR Signaling in Growth, Metabolism, and Disease. Cell, 168, 960-976.

8. Duvel, K., Yecies, J.L., Menon, S., Raman, P., Lipovsky, A.I., Souza, A.L., Triantafellow, E., Ma, Q., Gorski, R., Cleaver, S. et al. (2010) Activation of a metabolic gene regulatory network downstream of mTOR complex 1. Mol Cell, 39, 171-183.

9. Peterson, T.R., Sengupta, S.S., Harris, T.E., Carmack, A.E., Kang, S.A., Balderas, E., Guertin, D.A., Madden, K.L., Carpenter, A.E., Finck, B.N. et al. (2011) mTOR complex 1 regulates lipin 1 localization to control the SREBP pathway. Cell, 146, 408-420.

10. Sundqvist, A., Bengoechea-Alonso, M.T., Ye, X., Lukiyanchuk, V., Jin, J., Harper, J.W. and Ericsson, J. (2005) Control of lipid metabolism by phosphorylation-dependent degradation of the SREBP family of transcription factors by SCF(Fbw7). Cell Metab, 1, 379-391.

11. Welcker, M., Larimore, E.A., Swanger, J., Bengoechea-Alonso, M.T., Grim, J.E., Ericsson, J., Zheng, N. and Clurman, B.E. (2013) Fbw7 dimerization determines the specificity and robustness of substrate degradation. Genes Dev, 27, 2531-2536.

12. Li, S., Oh, Y.T., Yue, P., Khuri, F.R. and Sun, S.Y. (2016) Inhibition of mTOR complex 2 induces GSK3/FBXW7-dependent degradation of sterol regulatory element-binding protein 1 (SREBP1) and suppresses lipogenesis in cancer cells. Oncogene, 35, 642-650.

13. Comerford, S.A., Huang, Z., Du, X., Wang, Y., Cai, L., Witkiewicz, A.K., Walters, H., Tantawy, M.N., Fu, A., Manning, H.C. et al. (2014) Acetate dependence of tumors. Cell, 159, 1591-1602. 
14. Bulusu, V., Tumanov, S., Michalopoulou, E., van den Broek, N.J., MacKay, G., Nixon, C., Dhayade, S., Schug, Z.T., Vande Voorde, J., Blyth, K. et al. (2017) Acetate Recapturing by Nuclear Acetyl-CoA Synthetase 2 Prevents Loss of Histone Acetylation during Oxygen and Serum Limitation. Cell Rep, 18, 647-658.

15. Gao, X., Lin, S.H., Ren, F., Li, J.T., Chen, J.J., Yao, C.B., Yang, H.B., Jiang, S.X., Yan, G.Q., Wang, D. et al. (2016) Acetate functions as an epigenetic metabolite to promote lipid synthesis under hypoxia. Nat Commun, 7, 11960.

16. Yang, X.L., Khosravi-Far, R., Chang, H.Y. and Baltimore, D. (1997) Daxx, a novel Fasbinding protein that activates JNK and apoptosis. Cell, 89, 1067-1076.

18. Mahmud, I. and Liao, D. (2019) DAXX in cancer: phenomena, processes, mechanisms and regulation. Nucleic Acids Res, 47, 7734-7752.

19. Zhao, L.Y., Liu, J., Sidhu, G.S., Niu, Y., Liu, Y., Wang, R. and Liao, D. (2004) Negative regulation of p53 functions by Daxx and the involvement of MDM2. J Biol Chem, 279, 50566-50579.

20. Puto, L.A. and Reed, J.C. (2008) Daxx represses RelB target promoters via DNA methyltransferase recruitment and DNA hypermethylation. Genes Dev, 22, 998-1010.

21. Lewis, P.W., Elsaesser, S.J., Noh, K.M., Stadler, S.C. and Allis, C.D. (2010) Daxx is an H3.3specific histone chaperone and cooperates with ATRX in replication-independent chromatin assembly at telomeres. Proc Natl Acad Sci U S A, 107, 14075-14080.

22. Goldberg, A.D., Banaszynski, L.A., Noh, K.M., Lewis, P.W., Elsaesser, S.J., Stadler, S., Dewell, S., Law, M., Guo, X., Li, X. et al. (2010) Distinct factors control histone variant H3.3 localization at specific genomic regions. Cell, 140, 678-691.

23. Drane, P., Ouararhni, K., Depaux, A., Shuaib, M. and Hamiche, A. (2010) The deathassociated protein DAXX is a novel histone chaperone involved in the replicationindependent deposition of H3.3. Genes Dev, 24, 1253-1265.

24. Elsasser, S.J., Huang, H., Lewis, P.W., Chin, J.W., Allis, C.D. and Patel, D.J. (2012) DAXX envelops a histone H3.3-H4 dimer for H3.3-specific recognition. Nature, 491, 560-565.

25. Liu, C.P., Xiong, C., Wang, M., Yu, Z., Yang, N., Chen, P., Zhang, Z., Li, G. and Xu, R.M. (2012) Structure of the variant histone $\mathrm{H} 3.3-\mathrm{H} 4$ heterodimer in complex with its chaperone DAXX. Nat Struct Mol Biol, 19, 1287-1292.

26. Pan, W.W., Zhou, J.J., Liu, X.M., Xu, Y., Guo, L.J., Yu, C., Shi, Q.H. and Fan, H.Y. (2013) Death domain-associated protein DAXX promotes ovarian cancer development and chemoresistance. J Biol Chem, 288, 13620-13630.

27. Puto, L.A., Brognard, J. and Hunter, T. (2015) Transcriptional Repressor DAXX Promotes Prostate Cancer Tumorigenicity via Suppression of Autophagy. J Biol Chem, 290, 1540615420.

28. Benitez, J.A., Ma, J., D'Antonio, M., Boyer, A., Camargo, M.F., Zanca, C., Kelly, S., Khodadadi-Jamayran, A., Jameson, N.M., Andersen, M. et al. (2017) PTEN regulates glioblastoma oncogenesis through chromatin-associated complexes of DAXX and histone H3.3. Nat Commun, 8, 15223. 
29. Wang, Y., Stowe, R.L., Pinello, C.E., Tian, G., Madoux, F., Li, D., Zhao, L.Y., Li, J.L., Wang, Y., Wang, Y. et al. (2015) Identification of Histone Deacetylase Inhibitors with Benzoylhydrazide Scaffold that Selectively Inhibit Class I Histone Deacetylases. Chem Biol, 22, 273-284.

30. Mahmud, I. and Liao, D. (2015) Microarray gene expression profiling reveals potential mechanisms of tumor suppression by the class I HDAC-selective benzoylhydrazide inhibitors. Genom Data, 5, 257-259.

31. Bunse, L., Schumacher, T., Sahm, F., Pusch, S., Oezen, I., Rauschenbach, K., Gonzalez, M., Solecki, G., Osswald, M., Capper, D. et al. (2015) Proximity ligation assay evaluates IDH1R132H presentation in gliomas. J Clin Invest, 125, 593-606.

32. Waddell, A.R. and Liao, D. (2020) Assays for Validating Histone Acetyltransferase Inhibitors. J Vis Exp, e61289.

33. Subramanian, A., Tamayo, P., Mootha, V.K., Mukherjee, S., Ebert, B.L., Gillette, M.A., Paulovich, A., Pomeroy, S.L., Golub, T.R., Lander, E.S. et al. (2005) Gene set enrichment analysis: a knowledge-based approach for interpreting genome-wide expression profiles. Proc Natl Acad Sci U S A, 102, 15545-15550.

34. Pertea, M., Kim, D., Pertea, G.M., Leek, J.T. and Salzberg, S.L. (2016) Transcript-level expression analysis of RNA-seq experiments with HISAT, StringTie and Ballgown. Nat Protoc, 11, 1650-1667.

35. Kim, D., Langmead, B. and Salzberg, S.L. (2015) HISAT: a fast spliced aligner with low memory requirements. Nat Methods, 12, 357-360.

36. Pertea, M., Pertea, G.M., Antonescu, C.M., Chang, T.C., Mendell, J.T. and Salzberg, S.L. (2015) StringTie enables improved reconstruction of a transcriptome from RNA-seq reads. Nat Biotechnol, 33, 290-295.

37. Frazee, A.C., Pertea, G., Jaffe, A.E., Langmead, B., Salzberg, S.L. and Leek, J.T. (2015) Ballgown bridges the gap between transcriptome assembly and expression analysis. Nat Biotechnol, 33, 243-246.

38. Smyth, G.K. (2004) Linear models and empirical bayes methods for assessing differential expression in microarray experiments. Stat Appl Genet Mol Biol, 3, Article3.

39. Langmead, B. and Salzberg, S.L. (2012) Fast gapped-read alignment with Bowtie 2. Nat Methods, 9, 357-359.

40. Heinz, S., Benner, C., Spann, N., Bertolino, E., Lin, Y.C., Laslo, P., Cheng, J.X., Murre, C., Singh, H. and Glass, C.K. (2010) Simple combinations of lineage-determining transcription factors prime cis-regulatory elements required for macrophage and $B$ cell identities. Mol Cell, 38, 576-589.

41. Wang, Y., Viscarra, J., Kim, S.J. and Sul, H.S. (2015) Transcriptional regulation of hepatic lipogenesis. Nat Rev Mol Cell Biol, 16, 678-689.

42. Puto, L.A., Benner, C. and Hunter, T. (2015) The DAXX co-repressor is directly recruited to active regulatory elements genome-wide to regulate autophagy programs in a model of human prostate cancer. Oncoscience, 2, 362-372.

43. Horton, J.D., Shah, N.A., Warrington, J.A., Anderson, N.N., Park, S.W., Brown, M.S. and Goldstein, J.L. (2003) Combined analysis of oligonucleotide microarray data from transgenic and knockout mice identifies direct SREBP target genes. Proc Natl Acad Sci U $S A, 100,12027-12032$. 
44. Hoelper, D., Huang, H., Jain, A.Y., Patel, D.J. and Lewis, P.W. (2017) Structural and mechanistic insights into ATRX-dependent and -independent functions of the histone chaperone DAXX. Nat Commun, 8, 1193.

45. Currie, E., Schulze, A., Zechner, R., Walther, T.C. and Farese, R.V., Jr. (2013) Cellular fatty acid metabolism and cancer. Cell Metab, 18, 153-161.

46. Moon, S.H., Huang, C.H., Houlihan, S.L., Regunath, K., Freed-Pastor, W.A., Morris, J.P.t., Tschaharganeh, D.F., Kastenhuber, E.R., Barsotti, A.M., Culp-Hill, R. et al. (2019) p53 Represses the Mevalonate Pathway to Mediate Tumor Suppression. Cell, 176, 564-580 e519.

47. Escobar-Cabrera, E., Lau, D.K., Giovinazzi, S., Ishov, A.M. and McIntosh, L.P. (2010) Structural characterization of the DAXX N-terminal helical bundle domain and its complex with Rassf1C. Structure, 18, 1642-1653.

48. Bengoechea-Alonso, M.T. and Ericsson, J. (2007) SREBP in signal transduction: cholesterol metabolism and beyond. Curr Opin Cell Biol, 19, 215-222.

49. Porstmann, T., Santos, C.R., Griffiths, B., Cully, M., Wu, M., Leevers, S., Griffiths, J.R., Chung, Y.L. and Schulze, A. (2008) SREBP activity is regulated by mTORC1 and contributes to Akt-dependent cell growth. Cell Metab, 8, 224-236.

50. Michod, D., Bartesaghi, S., Khelifi, A., Bellodi, C., Berliocchi, L., Nicotera, P. and Salomoni, P. (2012) Calcium-dependent dephosphorylation of the histone chaperone DAXX regulates H3.3 loading and transcription upon neuronal activation. Neuron, 74, 122-135.

51. Martire, S., Gogate, A.A., Whitmill, A., Tafessu, A., Nguyen, J., Teng, Y.C., Tastemel, M. and Banaszynski, L.A. (2019) Phosphorylation of histone H3.3 at serine 31 promotes p300 activity and enhancer acetylation. Nat Genet, 51, 941-946.

52. Elsasser, S.J., Noh, K.M., Diaz, N., Allis, C.D. and Banaszynski, L.A. (2015) Histone H3.3 is required for endogenous retroviral element silencing in embryonic stem cells. Nature, 522, 240-244.

53. He, Q., Kim, H., Huang, R., Lu, W., Tang, M., Shi, F., Yang, D., Zhang, X., Huang, J., Liu, D. et al. (2015) The Daxx/Atrx Complex Protects Tandem Repetitive Elements during DNA Hypomethylation by Promoting H3K9 Trimethylation. Cell Stem Cell, 17, 273-286.

54. Yang, Y., Willis, T.L., Button, R.W., Strang, C.J., Fu, Y., Wen, X., Grayson, P.R.C., Evans, T., Sipthorpe, R.J., Roberts, S.L. et al. (2019) Cytoplasmic DAXX drives SQSTM1/p62 phase condensation to activate Nrf2-mediated stress response. Nat Commun, 10, 3759.

55. Mullen, P.J., Yu, R., Longo, J., Archer, M.C. and Penn, L.Z. (2016) The interplay between cell signalling and the mevalonate pathway in cancer. Nat Rev Cancer, 16, 718-731.

56. Riscal, R., Skuli, N. and Simon, M.C. (2019) Even Cancer Cells Watch Their Cholesterol! Mol Cell, 76, 220-231. 
752 Figure 1. DAXX is upregulated in $B C$ and transcriptomic profiling identifies a functional role for 753 DAXX in lipid metabolism. (A) Upregulation of DAXX mRNA expression in three major BC 754 subtypes compared to normal controls based on a TCGA dataset. (B) Increased DAXX protein 755 levels in three major BC subtypes compared to normal controls based on a CPTAC dataset. (C)

756 Validation of shRNA-mediated DAXX knockdown (KD) and the overexpression of WT DAXX 757 cDNA (OE) compared to cells with a control vector (CTL) in MDA-MB-231 cells by immunoblotting.

758 (D) Principal component analysis comparing transcriptomes of CTL, DAXX KD, and WT DAXX 759 OE cells. Each dot represents a sample. (E) Hierarchical clustering heatmap analysis of 760 differentially expressed genes showing 30 most differentially expressed genes. (F) Top 10 761 pathways identified using IPA as downregulated in DAXX KD but upregulated in DAXX OE cells.

762 (G) A heatmap of relative mRNA levels of DAXX along with select genes in the lipid metabolism

763 pathways. The red and blue groups refer to a high (red) or low level (blue) of mRNA expression

764 of the indicated genes according to combined expression scores in an individual tumor sample

765 from a TCGA BC dataset. (H) A Kaplan-Meier plot of the correlation between gene expression

766 levels of the select genes in panel $\mathbf{G}$ (the red and blue groups) and patient survival time.

768 Figure 2. DAXX promotes lipogenesis in cancer cells. (A) Principal component analysis 769 comparing lipidomes of MDA-MB-231 cells (CTL, DAXX KD and OE). Each dot represents a 770 sample $(n=6)$. (B) Hierarchical clustering heatmap analysis of the 60 most differentially expressed 771 lipid molecules in CTL, KD and OE MDA-MB-231 cells. (C) Significantly altered lipid pathways in 772 MDA-MB-231 cells with DAXX OE that were identified using the KEGG pathway library with an

773 FDR $<0.05$ and a pathway impact $>0.5$. The color and size of the circle denote $p$ value and 774 pathway impact respectively. The largest red circle indicates the most significantly affected 775 pathway. (D) An immunoblotting analysis of MDA-MB-468 cells with a control vector (CTL), DAXX 
776 shRNA (KD), and DAXX cDNA (OE). (E) Principal component analysis of lipidomes of CTL, KD

777 and OE MDA-MB-468 cells. Each dot represents a sample $(n=4)$. (F) Hierarchical clustering

778 heatmap analysis of top differentially changed lipid molecules in MDA-MB-468 cells. (G)

779 Significantly altered lipid pathways in MDA-MB-468 DAXX OE cells based on lipidome as in panel

780 C. The top 4 most altered pathways are labelled. (H) Impact of DAXX expression levels on

781 acetate-dependent de novo lipid synthesis using $\left[{ }^{14} \mathrm{C}\right]$-acetate labeling in the absence of serum

782 in the indicated cell lines with different levels of DAXX expression (CTL, KD or OE).

784 Figure 3. DAXX promotes tumor growth in vivo. (A and B) Cell lines derived from MDA-MB-231

785 or MDA-MB-468 stably transduced with a control vector (Control, CTL), DAXX shRNA (KD), or

786 WT DAXX cDNA (OE) were implanted into mammary fat pads of female NSG mice. Longitudinal 787 tumor volumes are plotted. Tumor images and weights at the endpoint are shown. (C) DAXX KD 788 and overexpression were maintained in vivo. Protein extracts from three representative xenograft 789 tumors were analyzed for DAXX protein levels using immunoblotting. HSP60 was detected as a

790 loading control. (D) Hierarchical clustering heatmap analysis of top glycerophospholipid 791 molecules that were differentially produced in MDA-MB-231 xenograft tumors with different levels

792 of DAXX. (E) Multivariate PCA of lipids shows distinct global lipid profiles in xenograft tumors 793 derived from control (red dots), DAXX KD (green dots), and OE (blue dots) MDA-MB-231 cells.

794 (F) Relative abundance of total triglycerides, cholesterol and derivatives in xenograft tumors 795 derived from MDA-MB-231 cell line panel as in (A). Box plots of the indicated lipid species are 796 shown. The $p$ values were calculated based on Student's t-test. *: $p<0.05 ;{ }^{* *}: p<0.01 .25 / 27-$ 797 HC: 25- or 27-hydroxycholesterol.

799 Figure 4 DAXX binds to SREBPs. (A) The endogenous DAXX and SREBP2 interact. MDA-MB800231 whole cell extracts were subjected to IP with a control (IgG) or an anti-DAXX antibody. The 801 immunoprecipitated SREBP2 and DAXX were detected. (B) Representative images of Proximity 
802 Ligation Assay (PLA) showing DAXX-SREBP2 interactions in MDA-MB-231 cells in the presence

803 or absence of serum. (C and $\mathbf{D}$ ) There are two independent binding sites in DAXX for mature

804 SREBP2. 293T cells were cotransfected with FLAG-SREBP2m (mature) and GFP (control) or an

805 indicated GFP-DAXX fusion construct. The cell lysates were subjected to anti-FLAG IP. Note

806 that the DAXX amino acid (aa) 129-190 construct is not recognized by the antibody used for

807 detecting DAXX in C (lane 2), which was detected with a GFP antibody (panel D, lane 2). The

808 endogenous DAXX in the input samples is denoted with an arrow in $\mathbf{C}$. In $\mathbf{D}$, the arrow points to

809 the GFP-DAXX 129-396 band, which accumulated at a relatively low level. HC: heavy chain. (E

810 and $\mathbf{F})$ The endogenous DAXX and SREBP1 interact. Total MDA-MB-231 cell extracts (E), the

811 cytoplasmic (C) or nuclear fraction (N) were subjected to IP was in panel A and the co-precipitated

812 SREBP1 and DAXX were detected. PCNA and HSP60 were detected as a marker of nuclear and

813 cytoplasmic fraction respectively in panel $\mathbf{F}$. (G) PLA images showing DAXX-SREBP1

814 interactions in MDA-MB-231 cells in the presence or absence of serum as in panel B. $(\mathbf{H})$

815 Cotransfection of FLAG-SREBP1a (mature) and the indicated GFP-DAXX fusion constructs, IP

816 and immunoblotting experiments were performed as in C. (I) Schematic drawing of DAXX-

817 SREBP interactions. The position of aa 327-335 within the DAXX HBD critical for the DAXX-

818 SREBP interactions is indicated. SIM: SUMO-interacting motif; 4HB: DAXX helical bundle; HBD:

819 histone-binding domain; PEST: proline, glutamic acid, serine, and threonine-rich sequence.

820 Numbers refer to aa residue positions in the DAXX protein. In panel $\mathbf{C}$, vertically sliced images

821 from the same gel are juxtaposed as indicated.

823 Figure 5. DAXX activates SREBP-mediated transcription and occupies the promoters of lipogenic

824 genes. (A) MDA-MB-231 cells were transfected with a luciferase reporter driven by a promoter 825 fragment from the SREBF2 gene along with mature SREBP2, SREBP1a, SREBP1c, or wt DAXX

826 cDNA as indicated. Dual luciferase assays were done. (B) ChIP-seq signal intensity heat maps

827 in MDA-MB-231 control and DAXX OE cell lines; signals are centralized to transcriptional start 
828 sites (TSS). (C) The genome-wide distribution of DAXX chromatin occupancy. (D) Motifs

829 enriched as determined by the DAXX ChIP-seq dataset of MDA-MB-231 DAXX OE cells. (E)

830 MDA-MB-231 cells stably expressing WT DAXX were subjected to ChIP with a control IgG and

831 an anti-DAXX antibody. The precipitated DNAs were subjected to qPCR with primers specific to

832 promoter regions of the indicated genes. (F) SREBP2 is critical for DAXX to bind lipogenic gene

833 promoters. MDA-MB-231 cells with a control vector or a SREBP2 shRNA vector were subjected

834 to ChIP with a control IgG, or an anti-DAXX antibody followed by qPCR with primers specific to

835 the indicated gene promoters.

837 Figure 6. SREBP2 knockdown impairs DAXX-mediated tumor growth. (A) Cells derived from

838 MDA-MB-231 cell line with a vector for a control, an SREBF2 shRNA, or SREBP2 (mature) cDNA

839 were subjected to RT-qPCR for assessing SREBP2 expression, and de novo lipogenesis assays

840 using $\left[{ }^{14} \mathrm{C}\right]$ acetate. (B) PCA of lipidomes in shControl and shSREBP2 cells. Each dot represents

841 a sample $(n=4)$. (C) Hierarchical clustering heatmap analysis demonstrates global lipid landscape

842 in shSREBP2 cells compared to shControl cells. (D) Hierarchical clustering heatmap analysis of

843 lipids including glycerolipid and glycerophospholipid molecules that were highly downregulated in

844 shSREBP2 cells compared to control cells. (E) MDA-MB-231-derived cells (Control, DAXX OE,

845 SREBP2 shRNA and mature SREBP2 OE) were xenografted into mammary fat pads of female

846 NSG mice. Tumor volumes were plotted against time. Representative images of dissected

847 tumors are shown. The final tumor weights are plotted. (F) Control or SREBF2 shRNA were

848 expressed in MDA-MB-231 cells with DAXX OE. The levels of the indicated proteins were

849 assessed by immunoblotting. (G) The indicated cells shown in panel F were xenografted into

850 mammary fat pads of female NSG mice. Tumor volumes were plotted against time.

851 Representative images of dissected tumors are shown. The final tumor weights are plotted. The

$852 p$ values were calculated (vs. control) based on Student's t-test. *: $p<0.05 ;{ }^{* *}: p<0.01$. 
854 Figure 7. The DAXX-SREBP interaction is critical for lipogenesis and tumor growth. (A) Relative 855 mRNA levels of DAXX in MDA-MB-231 cells expressing the WT or del 327-335 mutant cDNA of

856 DAXX as determined by RT-qPCR. (B) Protein levels of DAXX in control cells and those with

857 DAXX KD, WT and del 327-335 mutant cDNA of DAXX. (C) The DAXX del 327-337 mutant

858 impaired de novo lipogenesis. Serum-starved cells were labeled with $\left[{ }^{14} \mathrm{C}\right]$ acetate and total lipids

859 were isolated. Radioactivity was counted and normalized against total protein level. (D) PCA of

860 lipidomes in MDA-MB-231 cells expressing the del327-335 mutant and WT DAXX. Each dot

861 represents a sample $(n=4)$. (E) Hierarchical clustering heatmap analysis of global lipidomes in

862 cells expressing the del327-335 mutant and WT DAXX. (F) Hierarchical clustering heatmap

863 analysis of glycerolipid molecules that were highly differentially expressed between MDA-MB-231

864 cells with the del 327-335 mutant and wt DAXX. (G) Hierarchical clustering heatmap analysis of

865 glycerophospholipid molecules that were highly differentially expressed between MDA-MB-231

866 cells with the del 327-335 mutant and WT DAXX. $(\mathbf{H})$ Bar graphs of relative normalized

867 abundance of specific lipids in MDA-MB-231 cells expressing the del 327-335 mutant and WT

868 DAXX. (I) MDA-MB-231 cells expressing the del 327-335 mutant and WT DAXX were 869 xenografted into mammary fat pads of female NSG mice. Representative images of dissected 870 tumors are shown. The final tumor weights are plotted. (J) A cartoon depicting the importance 871 of DAXX-SREBP interaction for lipogenesis and tumorigenesis. The $p$ values were calculated (vs. 872 control) based on Student's t-test. *: $p<0.05 ;{ }^{* *}: p<0.01$. 


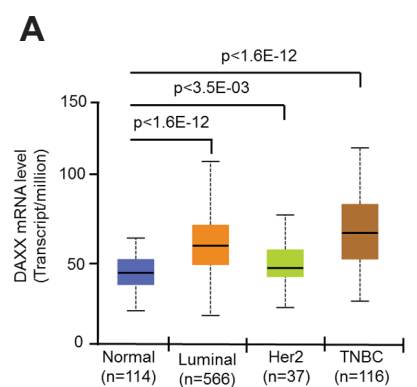

B
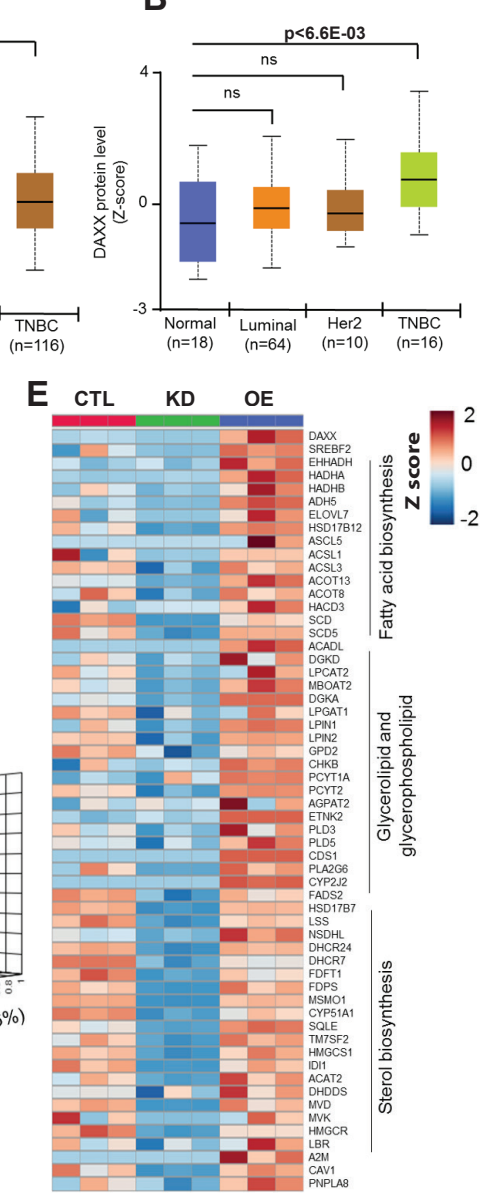

F Upstream Regulators in DAXX KD cells

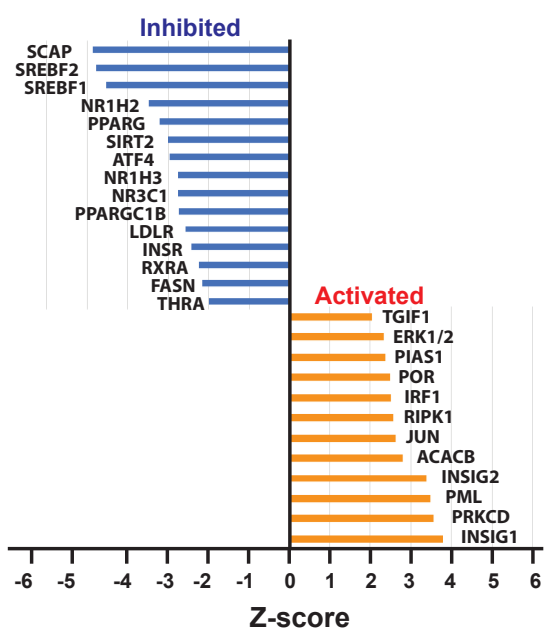

G

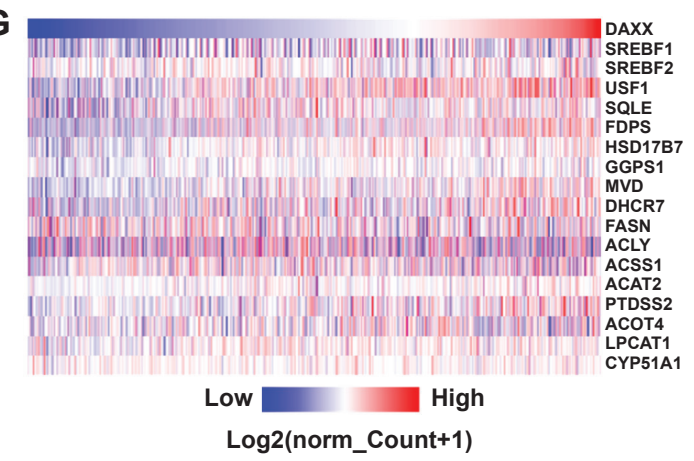

Upstream Regulators in DAXX OE cells

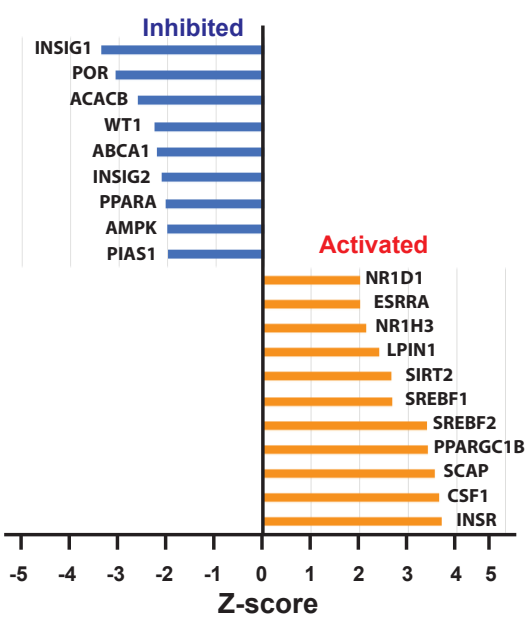

H TCGA breast cancer dataset

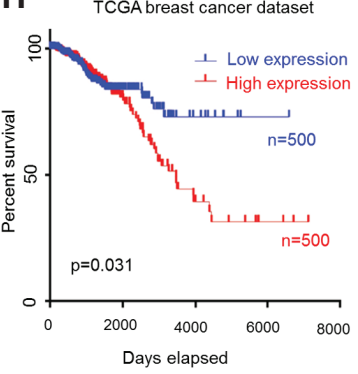

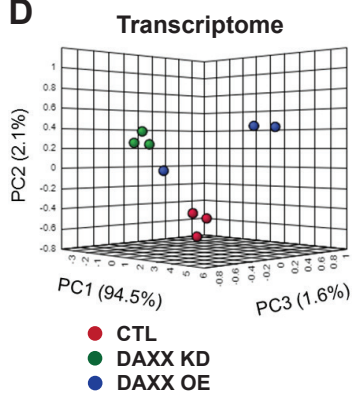

C

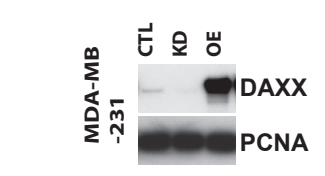

DAXX OE

Figure 1. DAXX is upregulated in $B C$ and transcriptomic profiling identifies a functional role for DAXX in lipid metabolism.

(A) Upregulation of DAXX mRNA expression in three major BC subtypes compared to normal controls based on a TCGA dataset. (B) Increased DAXX protein levels in three major BC subtypes compared to normal controls based on a CPTAC dataset. (C) Validation of shRNA-mediated DAXX knockdown (KD) and the overexpression of WT DAXX CDNA (OE) compared to cells with a control vector (CTL) in MDA-MB-231 cells by immunoblotting. (D) Principal component analysis comparing transcriptomes of CTL, DAXX KD, and WT DAXX OE cells. Each dot represents a sample. (E) Hierarchical clustering heatmap analysis of differentially expressed genes showing 30 most differentially expressed genes. (F) Top 10 pathways identified using IPA as downregulated in DAXX KD but upregulated in DAXX OE cells. (G) A heatmap of relative mRNA levels of DAXX along with select genes in the lipid metabolism pathways. The red and blue groups refer to a high (red) or low level (blue) of mRNA expression of the indicated genes according to combined expression scores in an individual tumor sample from a TCGA BC dataset. (H) A Kaplan-Meier plot of the correlation between gene expression levels of the select genes in panel $\mathrm{G}$ (the red and blue groups) and patient survival time. 
A

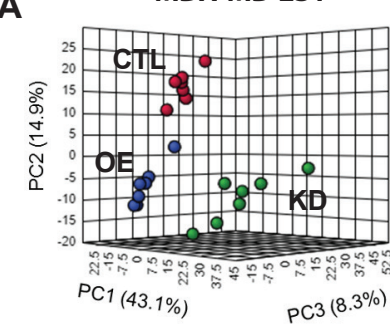

C

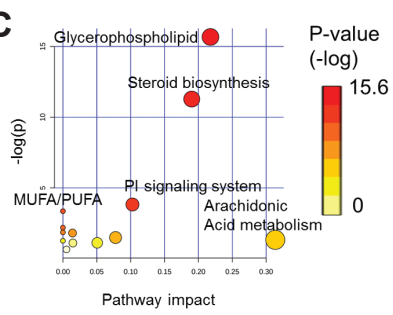

B

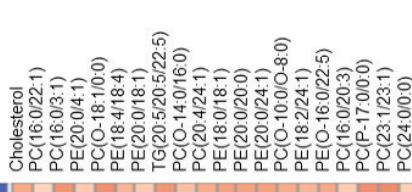

胥 $\log 2($ FC)

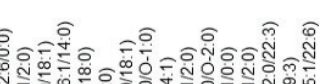

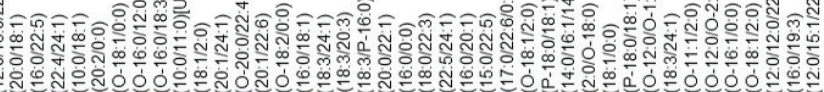

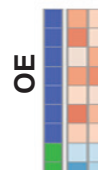

$\underline{x}$ ฉิ

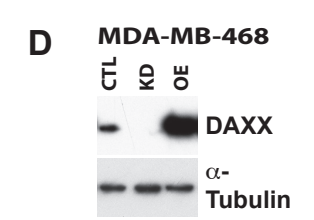

E

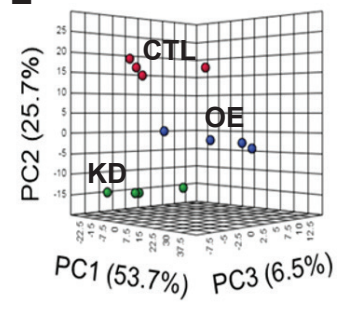

H

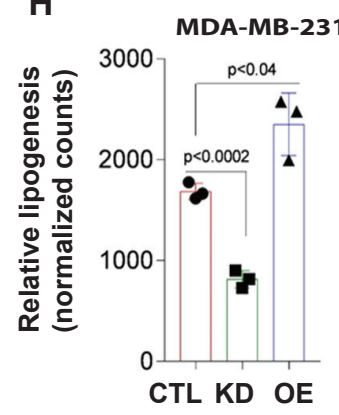

\section{$\mathbf{F}$}



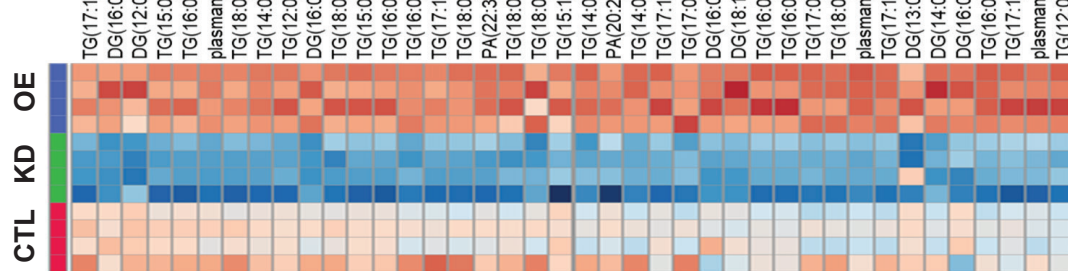

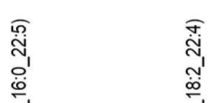

G
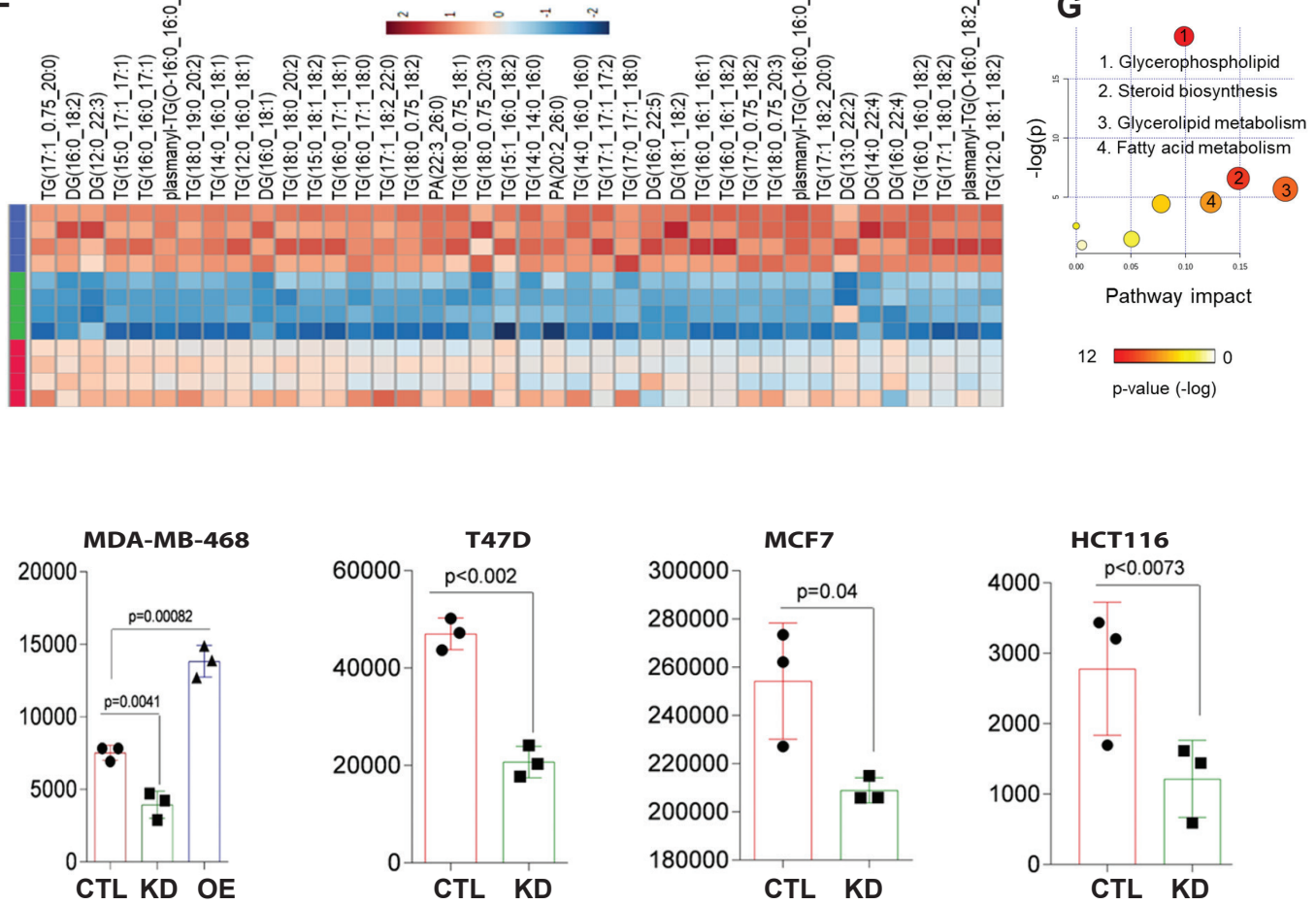

Figure 2. DAXX promotes lipogenesis in cancer cells.

(A) Principal component analysis comparing lipidomes of MDA-MB-231 cells (CTL, DAXX KD and OE). Each dot represents a sample $(n=6)$. (B) Hierarchical clustering heatmap analysis of the 60 most differentially expressed lipid molecules in CTL, KD and OE MDA-MB-231 cells. (C) Significantly altered lipid pathways in MDA-MB-231 cells with DAXX OE that were identified using the KEGG pathway library with an FDR $<0.05$ and a pathway impact $>0.5$. The color and size of the circle denote $p$ value and pathway impact respectively. The largest red circle indicates the most significantly affected pathway. (D) An immunoblotting analysis of MDA-MB-468 cells with a control vector (CTL), DAXX shRNA (KD), and DAXX CDNA (OE). (E) Principal component analysis of lipidomes of $C T L, K D$ and OE MDA-MB-468 cells. Each dot represents a sample $(n=4)$. (F) Hierarchical clustering heatmap analysis of top differentially changed lipid molecules in MDA-MB-468 cells. (G) Significantly altered lipid pathways in MDA-MB-468 DAXX OE cells based on lipidome as in panel C. The top 4 most altered pathways are labelled. (H) Impact of DAXX expression levels on acetate-dependent de novo lipid synthesis using $\left[{ }^{14} \mathrm{C}\right]$-acetate labeling in the absence of serum in the indicated cell lines with different levels of DAXX expression (CTL, KD or OE). 

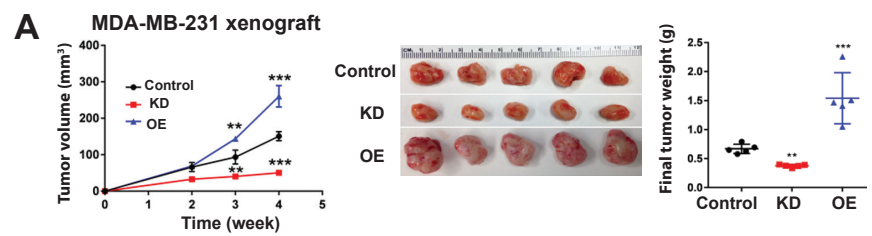

C MDA-MB-231 xenograft

B
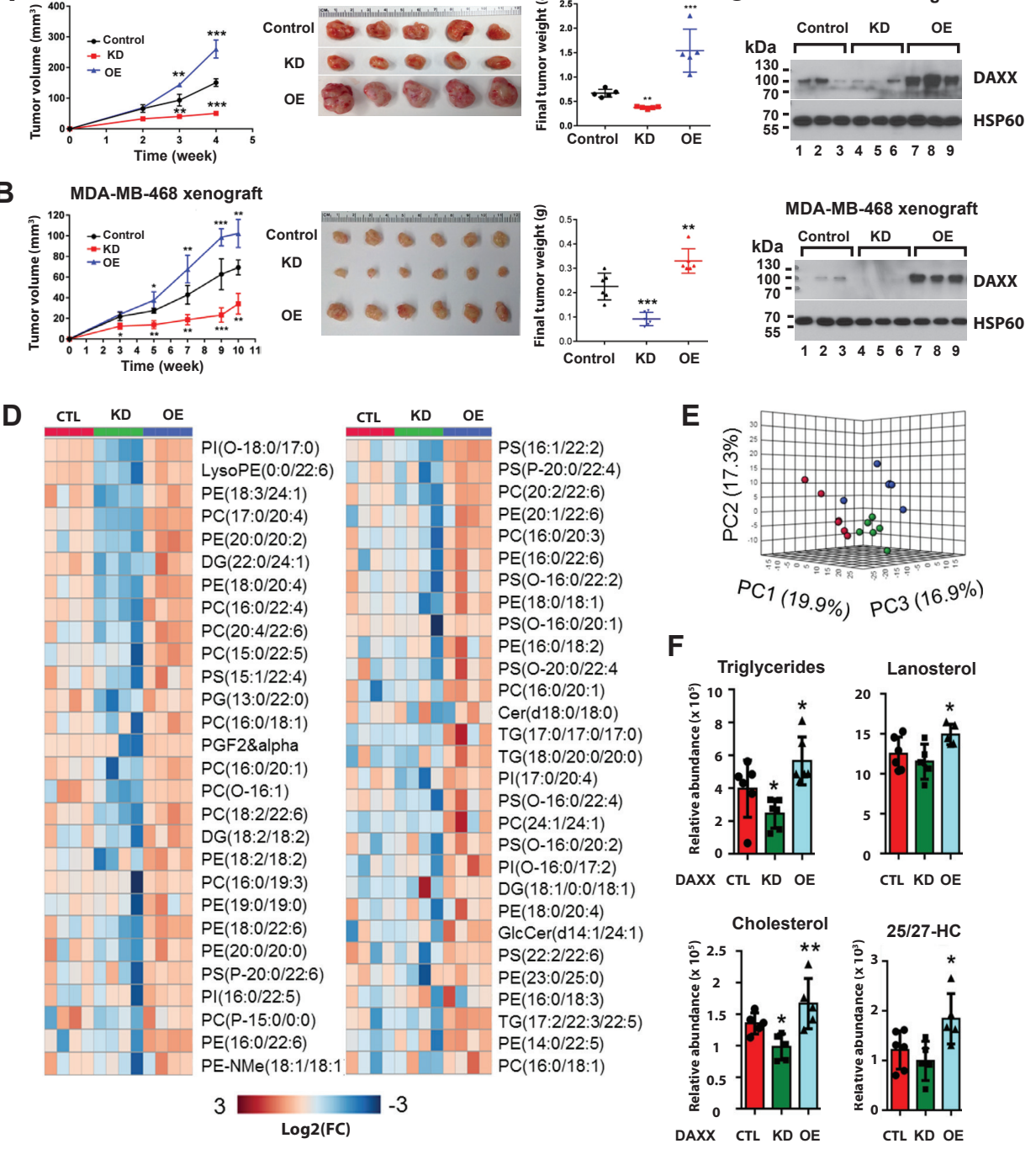

DAXX CTL KD OE

CTL KD OE

Figure 3. DAXX promotes tumor growth in vivo.

(A and B) Cell lines derived from MDA-MB-231 or MDA-MB-468 stably transduced with a control vector (Control, CTL), DAXX shRNA (KD), or WT DAXX CDNA (OE) were implanted into mammary fat pads of female NSG mice. Longitudinal tumor volumes are plotted. Tumor images and weights at the endpoint are shown. (C) DAXX KD and overexpression were maintained in vivo. Protein extracts from three representative xenograft tumors were analyzed for DAXX protein levels using immunoblotting. HSP60 was detected as a loading control. (D) Hierarchical clustering heatmap analysis of top glycerophospholipid molecules that were differentially produced in MDA-MB-231 xenograft tumors with different levels of DAXX. (E) Multivariate PCA of lipids shows distinct global lipid profiles in xenograft tumors derived from control (red dots), DAXX KD (green dots), and OE (blue dots) MDA-MB-231 cells. (F) Relative abundance of total triglycerides, cholesterol and derivatives in xenograft tumors derived from MDA-MB-231 cell line panel as in (A). Box plots of the indicated lipid species are shown. The $p$ values were calculated based on Student's t-test. ${ }^{*}: p<0.05 ;{ }^{* *}: p<0.01 .25 / 27-H C: 25$ - or 27-hydroxycholesterol. 
bioRxiv preprint doi: https://doi.org/10.1101/2020.12.31.424997; this version posted January 3, 2021. The copyright holder for this preprint (which was not certified by peer review) is the author/funder. All rights reserved. No reuse allowed without permission.

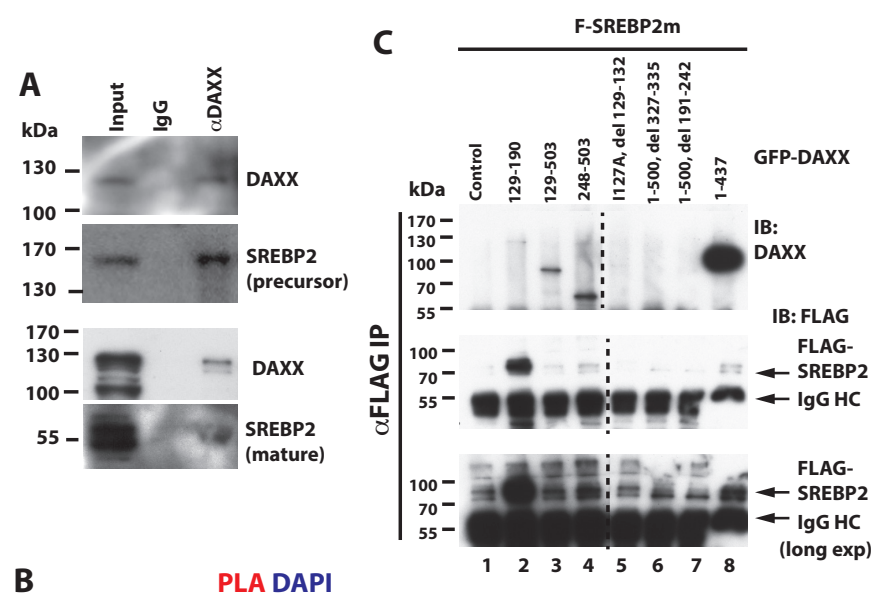

B
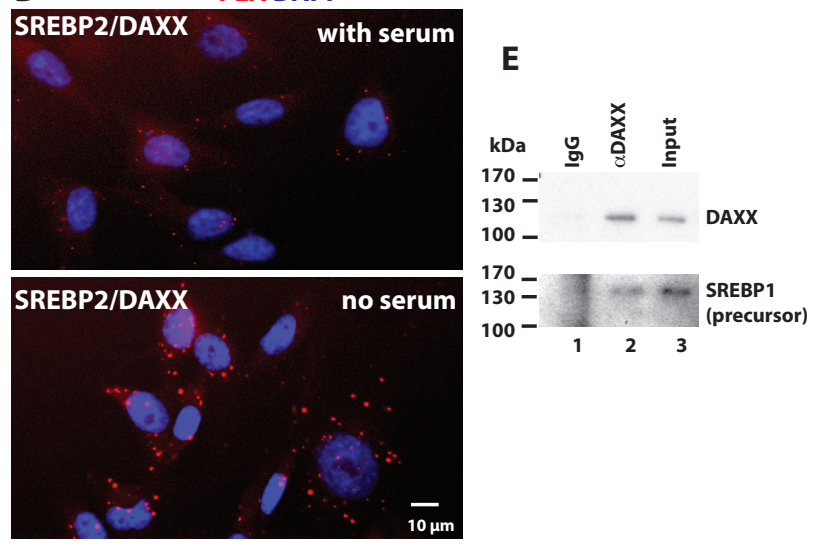

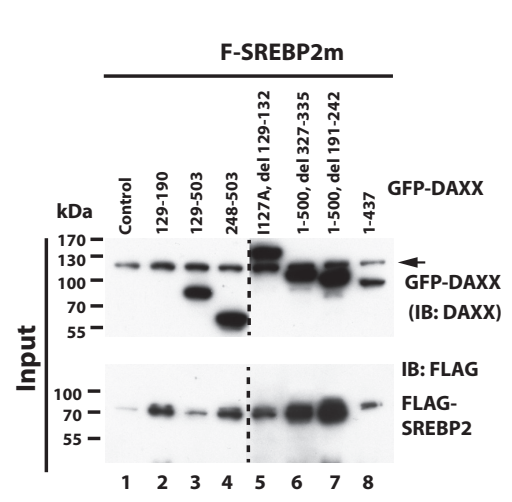

D

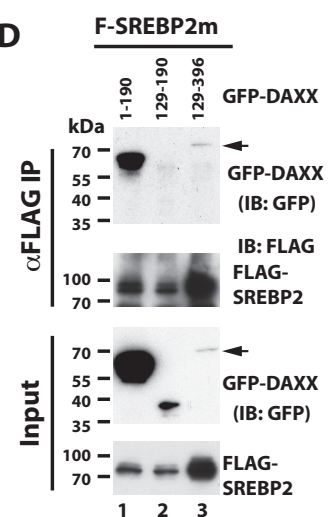

PLA DAPI
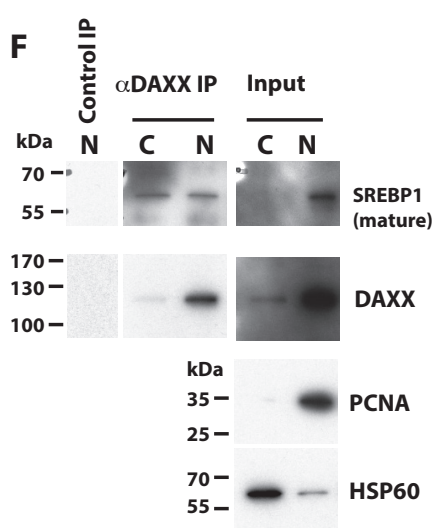

G
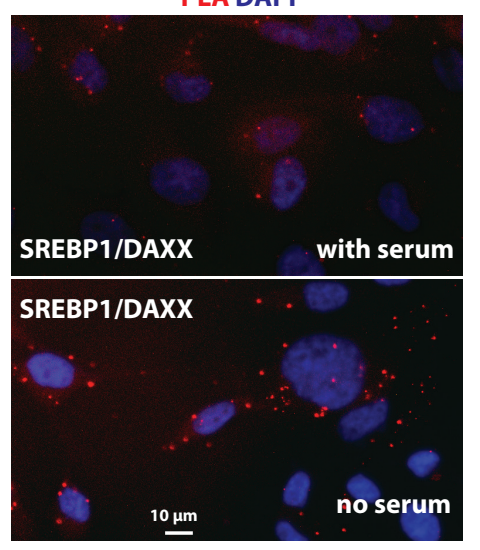

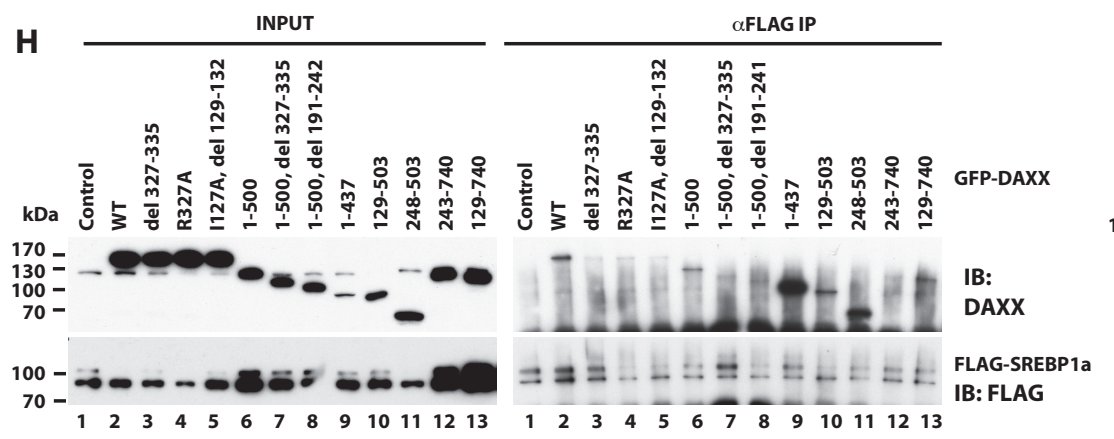

I

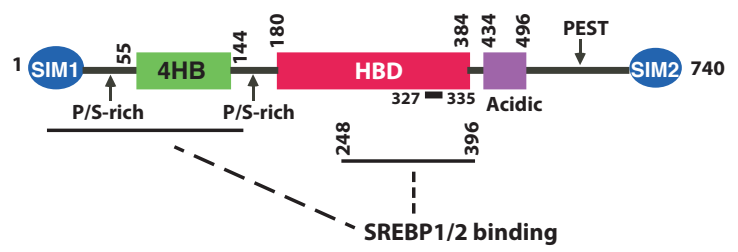

Figure 4 DAXX binds to SREBPs.

(A) The endogenous DAXX and SREBP2 interact. MDA-MB-231 whole cell extracts were subjected to IP with a control (IgG) or an anti-DAXX antibody. The immunoprecipitated SREBP2 and DAXX were detected. (B) Representative images of Proximity Ligation Assay (PLA) showing DAXX-SREBP2 interactions in MDA-MB-231 cells in the presence or absence of serum. (C and $\mathbf{D})$ There are two independent binding sites in DAXX for mature SREBP2. 293T cells were cotransfected with FLAG-SREBP2m (mature) and GFP (control) or an indicated GFP-DAXX fusion construct. The cell lysates were subjected to anti-FLAG IP. Note that the DAXX amino acid (aa) 129-190 construct is not recognized by the antibody used for detecting DAXX in C (lane 2), which was detected with a GFP antibody (panel D, lane 2). The endogenous DAXX in the input samples is denoted with an arrow in $\mathbf{C}$. In D, the arrow points to the GFP-DAXX 129-396 band, which accumulated at a relatively low level. HC: heavy chain. (E and F) The endogenous DAXX and SREBP1 interact. Total MDA-MB-231 cell extracts (E), the cytoplasmic (C) or nuclear fraction (N) were subjected to IP was in panel A and the co-precipitated SREBP1 and DAXX were detected. PCNA and HSP60 were detected as a marker of nuclear and cytoplasmic fraction respectively in panel F. (G) PLA images showing DAXX-SREBP1 interactions in MDA-MB-231 cells in the presence or absence of serum as in panel B. (H) Cotransfection of FLAG-SREBP1a (mature) and the indicated GFP-DAXX fusion constructs, IP and immunoblotting experiments were performed as in C. (I) Schematic drawing of DAXX-SREBP interactions. The position of aa 327-335 within the DAXX HBD critical for the DAXX-SREBP interactions is indicated. SIM: SUMO-interacting motif; 4HB: DAXX helical bundle; HBD: histone-binding domain; PEST: proline, glutamic acid, serine, and threonine-rich sequence. Numbers refer to aa residue positions in the DAXX protein. In panel C, vertically sliced images from the same gel are juxtaposed as indicated. 
E
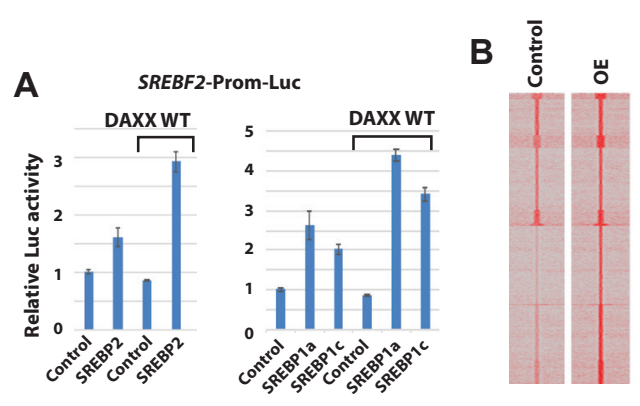

C

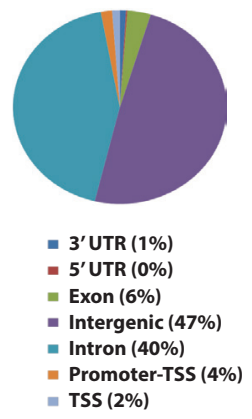

D ATGGTAGATCCA NF-kB1

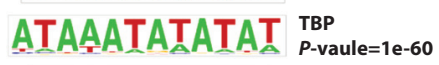
CCTTCAGAGGGT ${ }_{\substack{\text { p.vau } \\ \text { P. }}}$ ATCGCCTTGAA

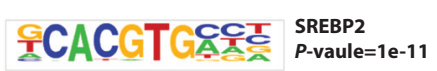
है SGCTCACGTGA Usfir GACTTGGTGCCC P.vaule=1e-38 $_{\text {HII }}^{\text {Hal }}$

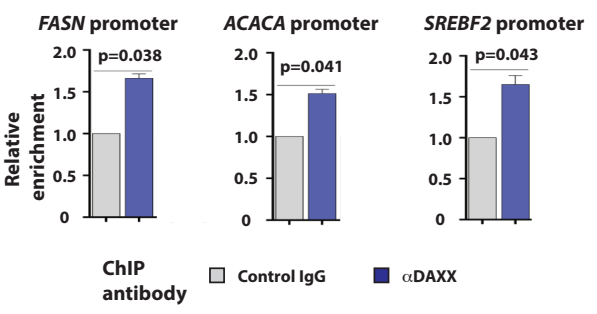

$\mathbf{F}$

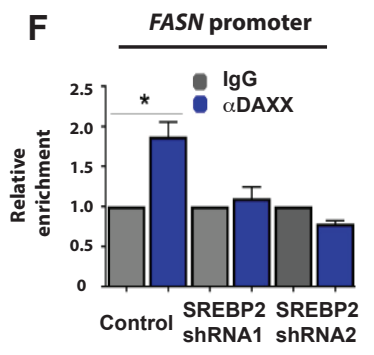

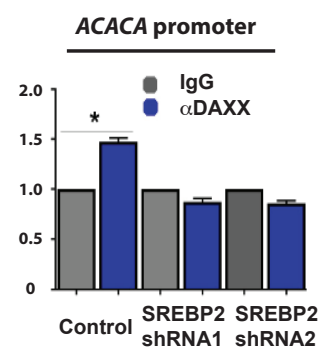

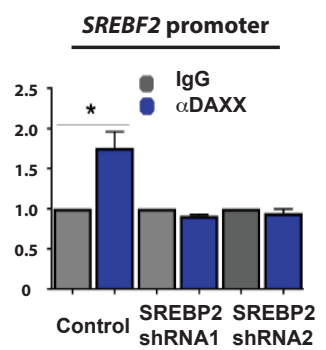

Figure 5. DAXX activates SREBP-mediated transcription and occupies the promoters of lipogenic genes.

(A) MDA-MB-231 cells were transfected with a luciferase reporter driven by a promoter fragment from the SREBF2 gene along with mature SREBP2, SREBP1a, SREBP1c, or wt DAXX CDNA as indicated. Dual luciferase assays were done. (B) ChIP-seq signal intensity heat maps in MDA-MB-231 control and DAXX OE cell lines; signals are centralized to transcriptional start sites (TSS). (C) The genome-wide distribution of DAXX chromatin occupancy. (D) Motifs enriched as determined by the DAXX ChIP-seq dataset of MDA-MB-231 DAXX OE cells. (E) MDA-MB-231 cells stably expressing WT DAXX were subjected to ChIP with a control IgG and an anti-DAXX antibody. The precipitated DNAs were subjected to qPCR with primers specific to promoter regions of the indicated genes. (F) SREBP2 is critical for DAXX to bind lipogenic gene promoters. MDA-MB-231 cells with a control vector or a SREBP2 shRNA vector were subjected to ChIP with a control lgG, or an anti-DAXX antibody followed by qPCR with primers specific to the indicated gene promoters. 
A

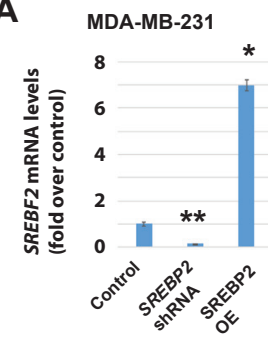

B

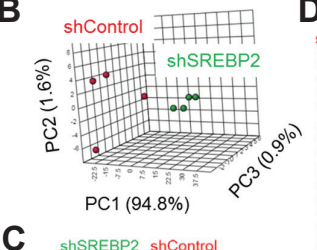

C

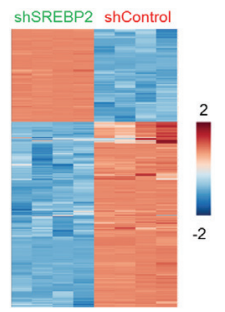

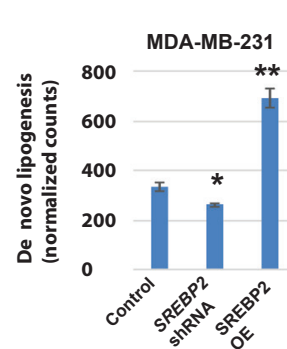

D

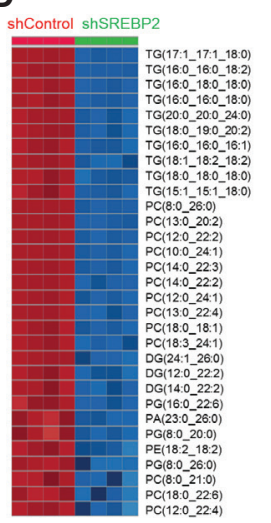

E
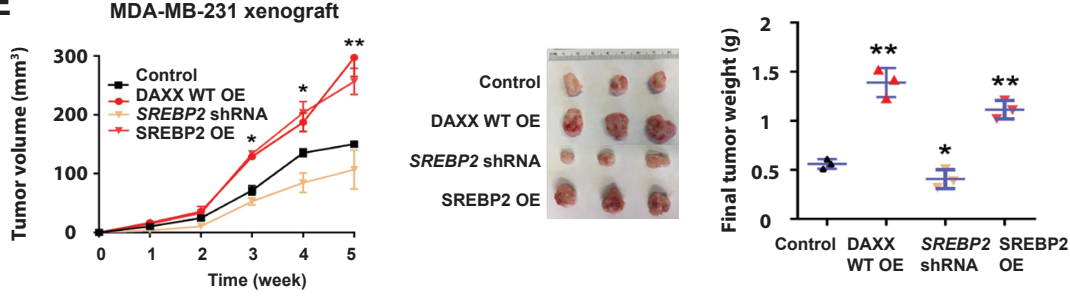

$\mathbf{F}$

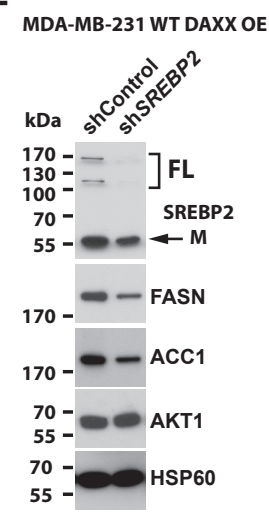

G

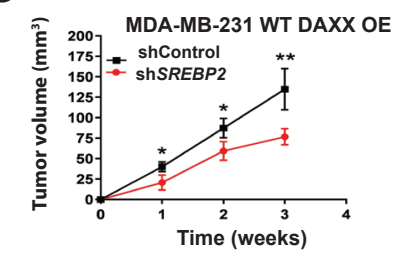

MDA-MB-231 WT DAXX OE

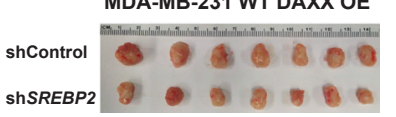

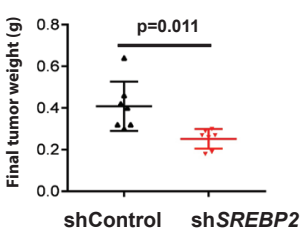

Figure 6. SREBP2 knockdown impairs DAXX-mediated tumor growth.

(A) Cells derived from MDA-MB-231 cell line with a vector for a control, an SREBF2 shRNA, or SREBP2 (mature) cDNA were subjected to RT-qPCR for assessing SREBP2 expression, and de novo lipogenesis assays using $\left[{ }^{14} \mathrm{C}\right]$ acetate. (B) PCA of lipidomes in shControl and shSREBP2 cells. Each dot represents a sample $(n=4)$. (C) Hierarchical clustering heatmap analysis demonstrates global lipid landscape in shSREBP2 cells compared to shControl cells. (D) Hierarchical clustering heatmap analysis of lipids including glycerolipid and glycerophospholipid molecules that were highly downregulated in shSREBP2 cells compared to control cells. (E) MDA-MB-231-derived cells (Control, DAXX OE, SREBP2 shRNA and mature SREBP2 OE) were xenografted into mammary fat pads of female NSG mice. Tumor volumes were plotted against time. Representative images of dissected tumors are shown. The final tumor weights are plotted. (F) Control or SREBF2 shRNA were expressed in MDA-MB-231 cells with DAXX OE. The levels of the indicated proteins were assessed by immunoblotting. (G) The indicated cells shown in panel $\mathrm{F}$ were xenografted into mammary fat pads of female NSG mice. Tumor volumes were plotted against time. Representative images of dissected tumors are shown. The final tumor weights are plotted. The $p$ values were calculated (vs. control) based on Student's t-test. *: $p<0.05 ;{ }^{* *}: p<0.01$. 

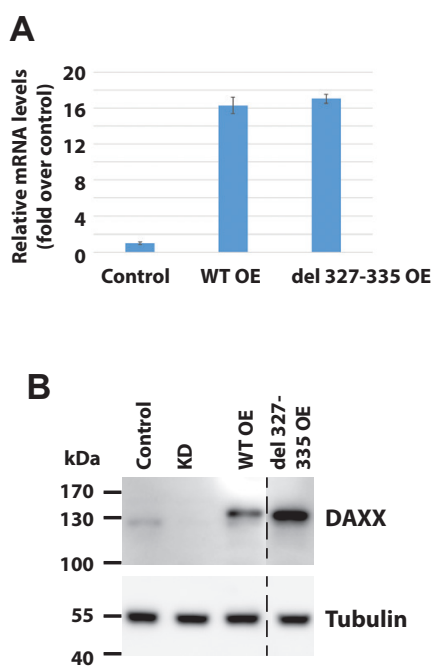

C

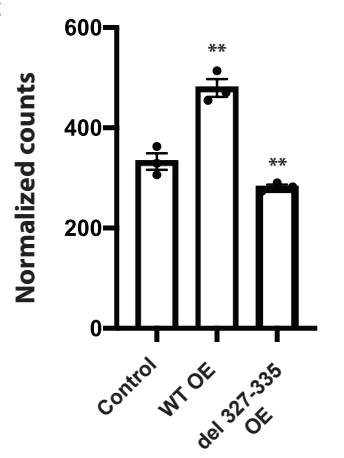

D

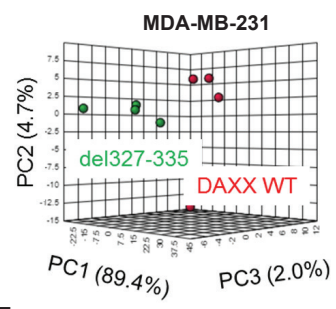

E

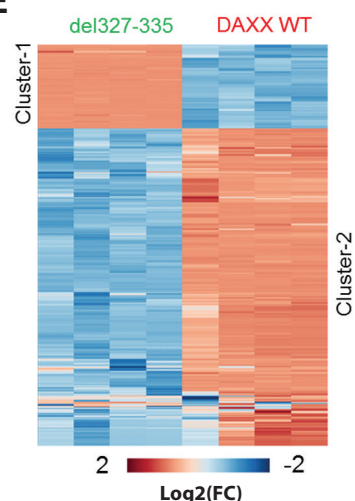

$\mathbf{F}$

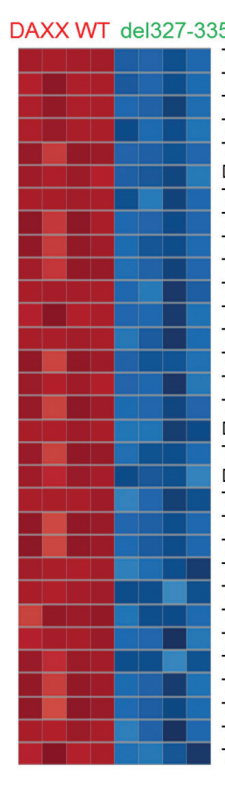

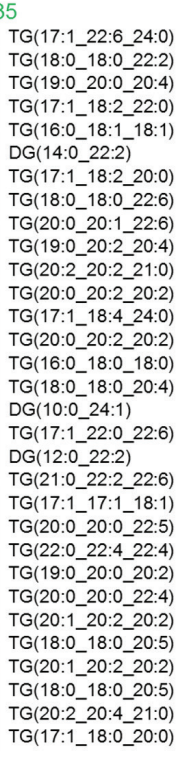

G

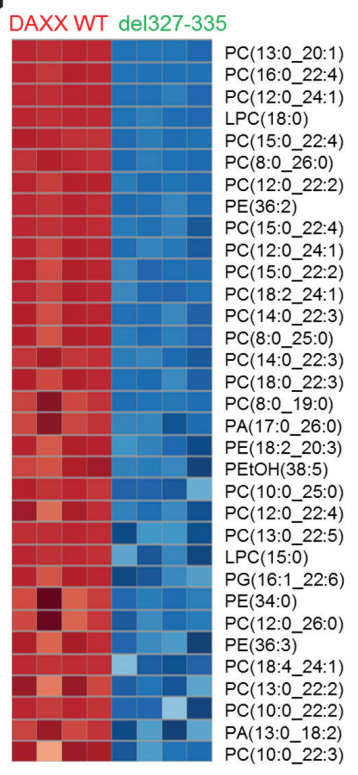

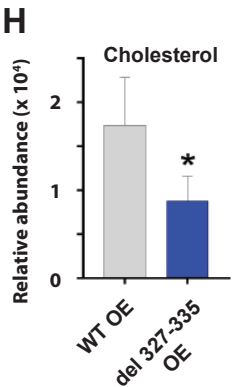
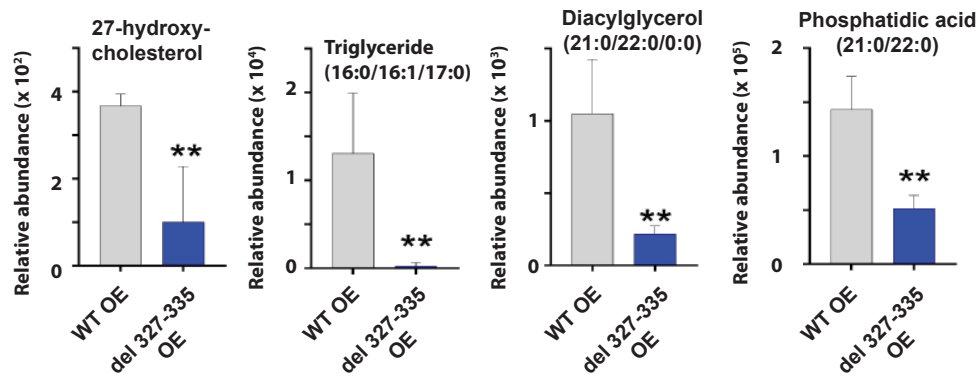

I
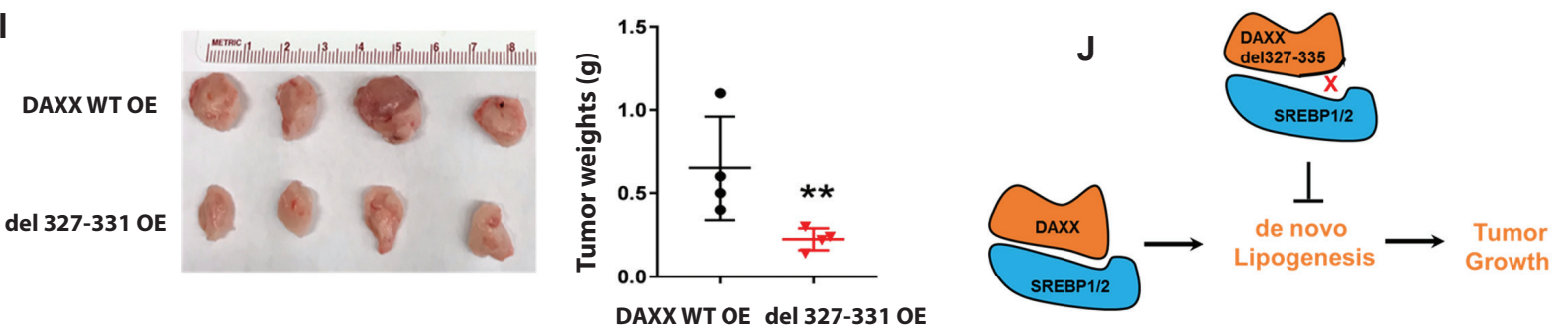

Figure 7. The DAXX-SREBP interaction is critical for lipogenesis and tumor growth.

(A) Relative mRNA levels of DAXX in MDA-MB-231 cells expressing the WT or del 327-335 mutant CDNA of DAXX as determined by RT-qPCR. (B) Protein levels of DAXX in control cells and those with DAXX KD, WT and del 327-335 mutant CDNA of DAXX. (C) The DAXX del 327-337 mutant impaired de novo lipogenesis. Serum-starved cells were labeled with $\left[{ }^{14} \mathrm{C}\right]$ acetate and total lipids were isolated. Radioactivity was counted and normalized against total protein level. (D) PCA of lipidomes in MDA-MB-231 cells expressing the del327-335 mutant and WT DAXX. Each dot represents a sample $(n=4)$. (E) Hierarchical clustering heatmap analysis of global lipidomes in cells expressing the del327-335 mutant and WT DAXX. (F) Hierarchical clustering heatmap analysis of glycerolipid molecules that were highly differentially expressed between MDA-MB-231 cells with the del 327-335 mutant and wt DAXX. (G) Hierarchical clustering heatmap analysis of glycerophospholipid molecules that were highly differentially expressed between MDA-MB-231 cells with the del 327-335 mutant and WT DAXX. (H) Bar graphs of relative normalized abundance of specific lipids in MDA-MB-231 cells expressing the del 327-335 mutant and WT DAXX. (I) MDA-MB-231 cells expressing the del 327-335 mutant and WT DAXX were xenografted into mammary fat pads of female NSG mice. Representative images of dissected tumors are shown. The final tumor weights are plotted. (J) A cartoon depicting the importance of DAXX-SREBP interaction for lipogenesis and tumorigenesis. The $\mathrm{p}$ values were calculated (vs. control) based on Student's t-test. * $p<0.05 ;{ }^{* *}: p<0.01$. 


\section{Mahmud et al. Supplementary Figures and Tables}

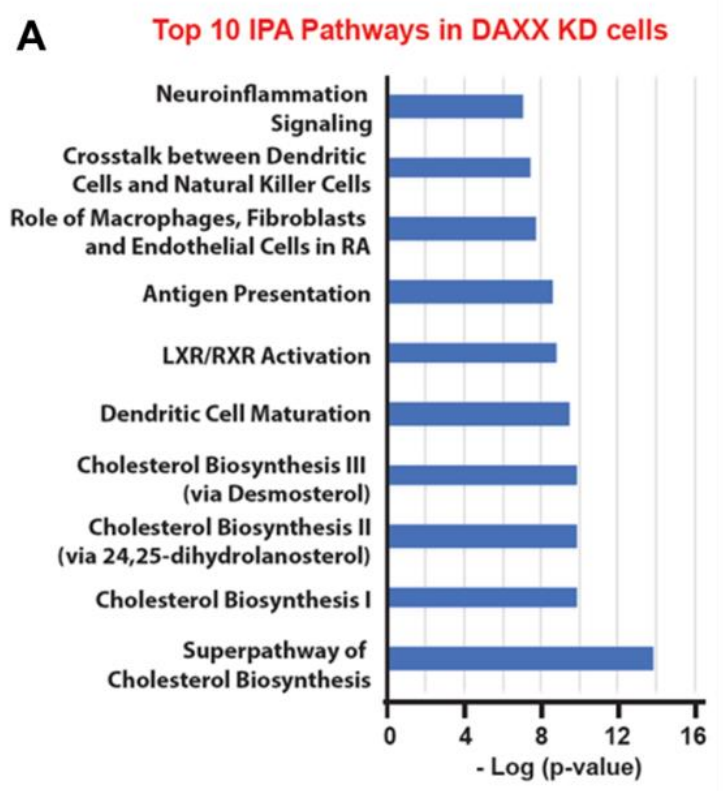

B GSEA: Cholesterol biosynthesis

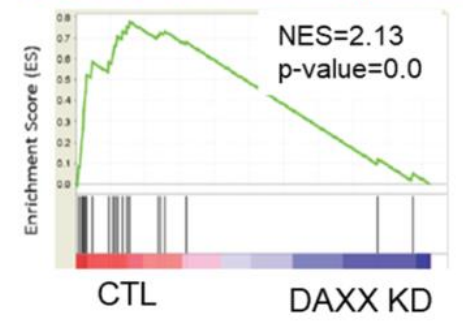

GSEA: Cholesterol biosynthesis

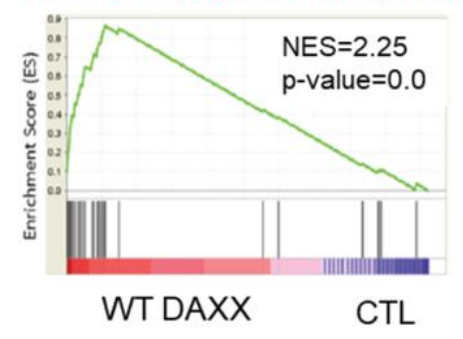

C

GSEA: Glycerophospholipid

GSEA: Fatty acid metabolism
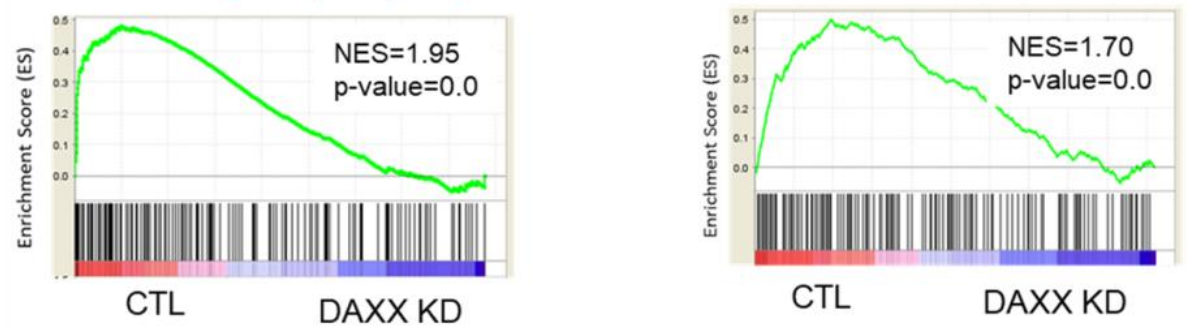

CTL

DAXX KD

GSEA: Glycerophospholipid

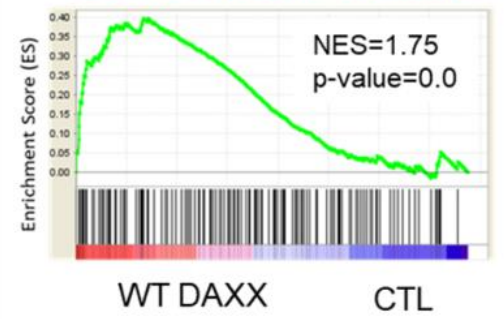

GSEA: Fatty acid metabolism

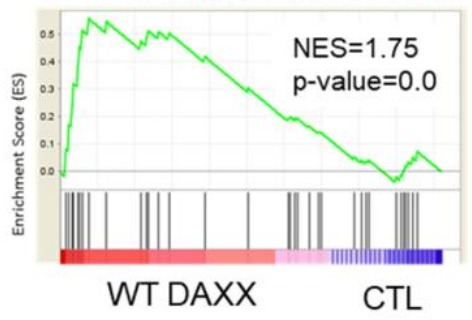

\section{Supplementary Figure S1. DAXX expression correlates with de novo lipogenesis pathway.}

(A) Ingenuity pathway analysis (IPA) using differentially expressed genes in DAXX KD cells compared to CTL cells identifies de novo lipogenesis as the most perturbed canonical pathways.

(B) Gene set enrichment analyses (GSEA) shows downregulation or upregulation of genes in the cholesterol biosynthesis in MDA-MB-231 cells with DAXX KD or WT DAXX OE, respectively. The KEGG and Reactome genesets were used for the GSEA plots. 
bioRxiv preprint doi: https://doi.org/10.1101/2020.12.31.424997; this version posted January 3, 2021. The copyright holder for this preprint (which was not certified by peer review) is the author/funder. All rights reserved. No reuse allowed without permission.

(C) Gene set enrichment analyses (GSEA) shows downregulation or upregulation of genes in the fatty acid, glycerophospholipid, and glycerolipid metabolism in MDA-MB-231 cells with DAXX KD or WT DAXX OE, respectively. The KEGG and Reactome genesets were used for the GSEA plots. 

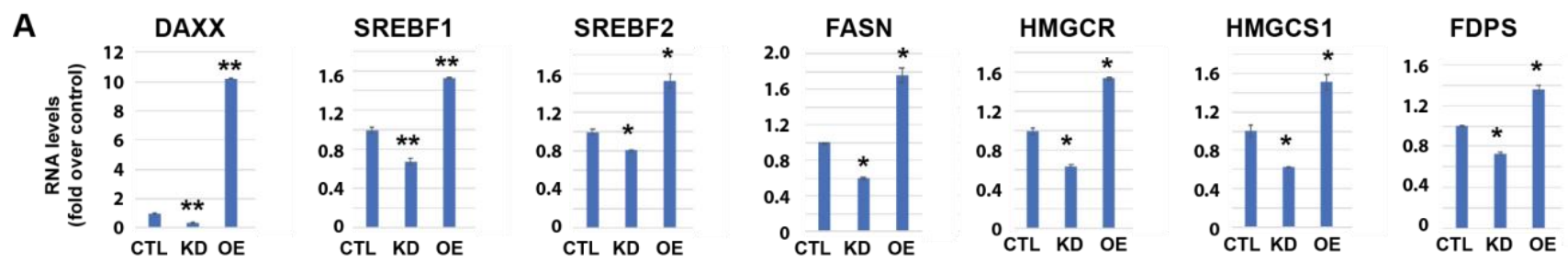

B MDA-MB-231
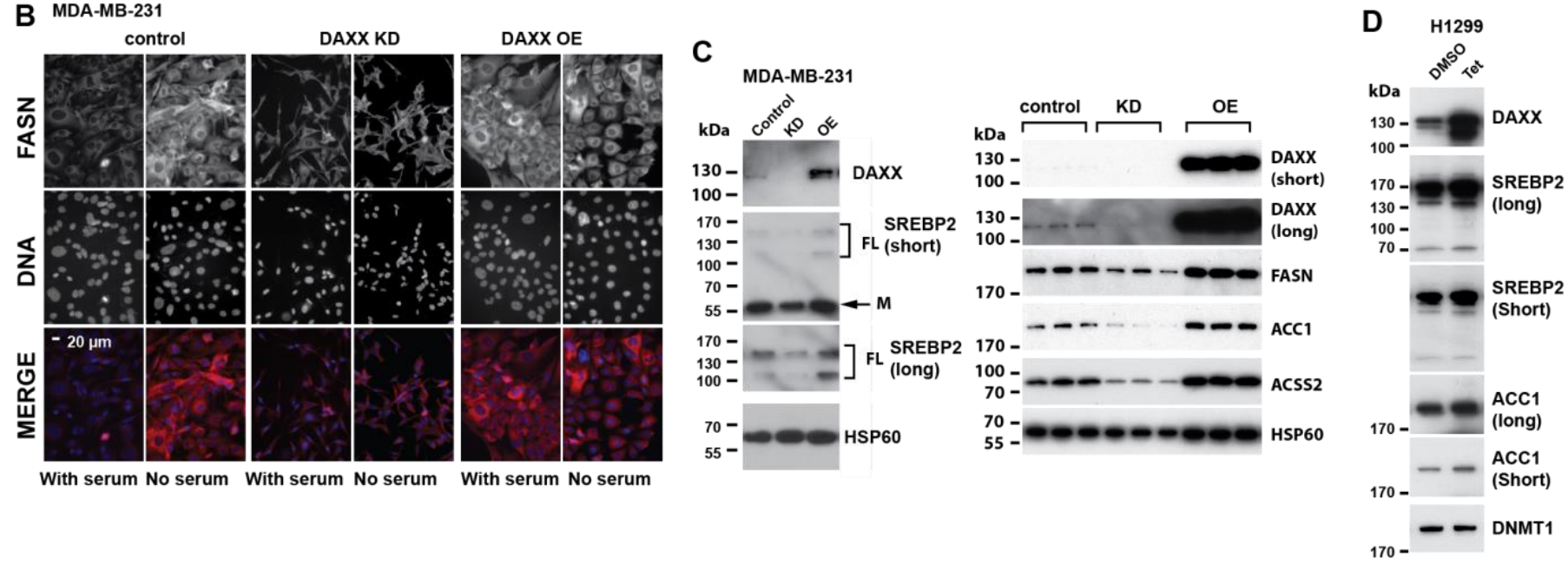

\section{Supplementary Figure S2. DAXX promotes lipogenic gene expression.}

(A) Total RNAs were isolated from MDA-MB-231 cells stably transfected with a control vector (CTL), a DAXX shRNA (KD), the WT DAXX cDNA (OE) and subjected to RT-qPCR analysis. The mRNA levels of the indicated genes were normalized against that of ACTB. Data are shown as mean of fold-changes vs. control (CTL) \pm SEM $(n=3) . *$ : $p<0.05, * *: p<0.01$ (t-test vs $C T L)$.

(B) MDA-MB-231-derived cells were cultured in the presence of serum or serum-starved for 24 hours. The cells were then fixed and stained with an anti-FASN polyclonal antibody (red) and counterstained with DAPI for visualizing nuclei (blue). The cells were imaged using a fluorescence microscope. All images were captured with the same duration of light exposure for the red or blue channel.

(C) Immunoblotting analysis of cell extracts of the MDA-MB-231-derived cell lines with antibodies against the indicated proteins.

(D) Immunoblotting analysis of cell extracts of the H1299 cells expressing tetracycline (Tet)-inducible wt DAXX in the presence of control (DMSO) or Tet with antibodies against the indicated proteins. 
PC3

(Puto et al., 2015)

A

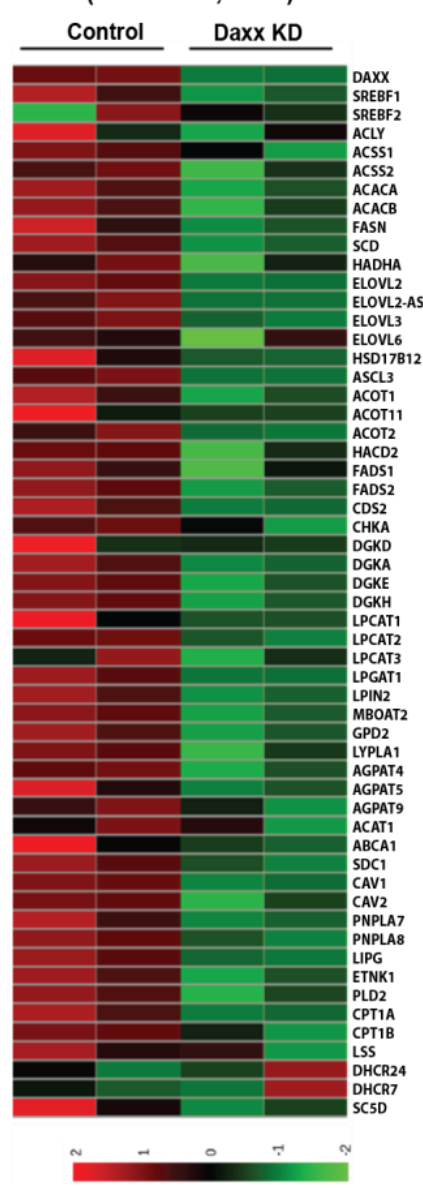

B $\mathrm{mESC}$

B (Hoelper et al., 2017)

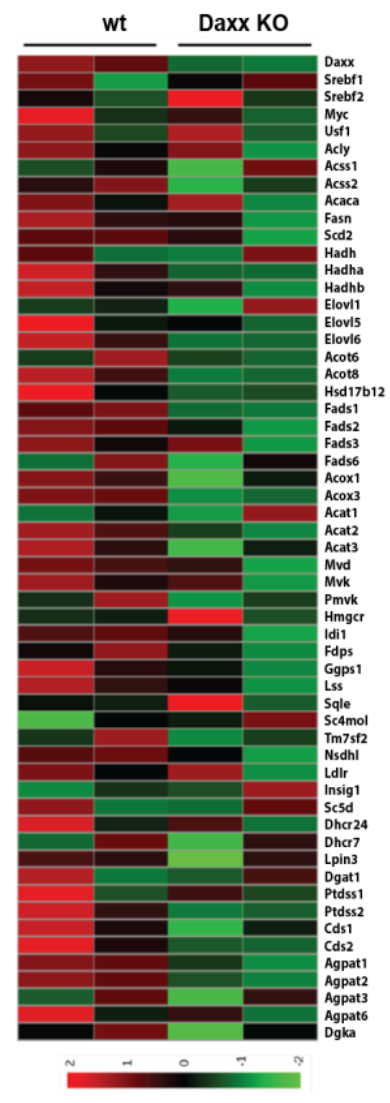

C Liver tissues of transgenic mice

(Horton et al., 2003)

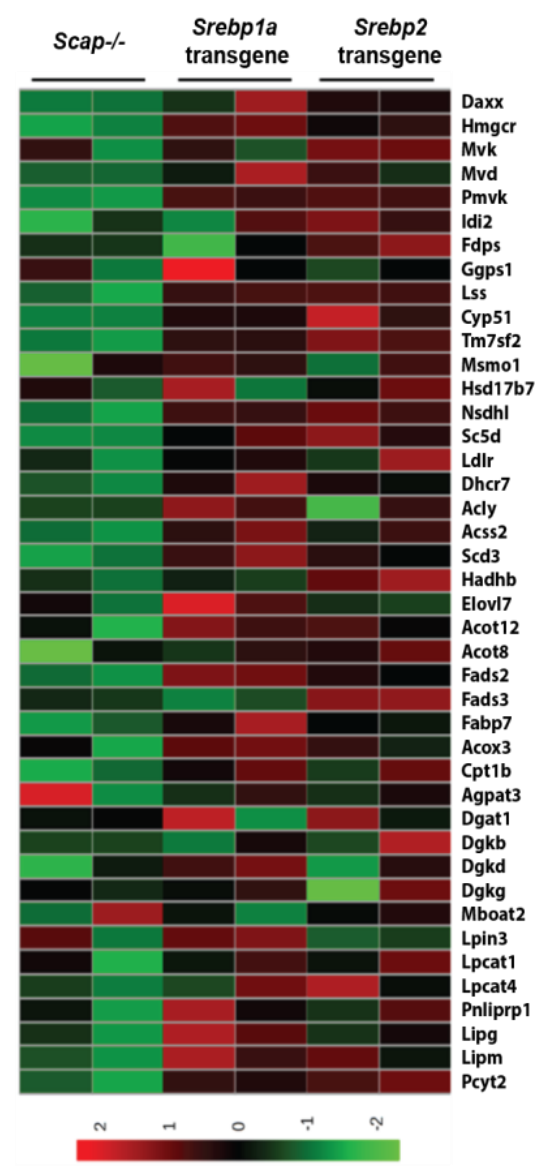

\section{Supplementary Figure S3. DAXX, SREBP1 and SREBP2 are key regulators for lipogenic gene expression.}

(A) Heatmap of the indicated lipogenic genes in control and DAXX KD cells of the human prostate cancer PC3 cell line.

(B) Heatmap of the indicated lipogenic genes in wt and Daxx KO cells of the mouse embryonic stem cells (mESC).

(C) Heatmap of the indicated lipogenic genes in liver tissues from mice with Scap deletion (Scap-/-), the nuclear form of Srebp1a or Srebp2 transgene. 
bioRxiv preprint doi: https://doi.org/10.1101/2020.12.31.424997; this version posted January 3, 2021. The copyright holder for this preprint (which was not certified by peer review) is the author/funder. All rights reserved. No reuse allowed without permission.

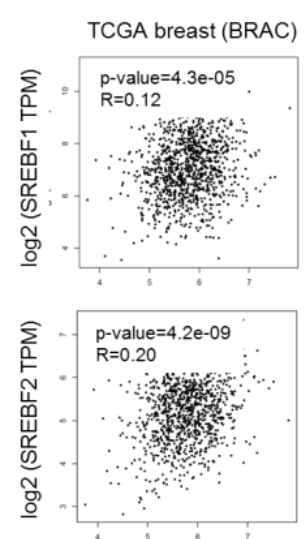

$\log 2(D A X X$ TPM) TCGA Glioma (GBM)
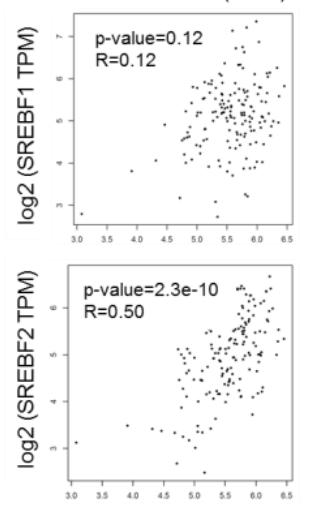

$\log 2$ (DAXX TPM)

TCGA Liver (LIHC)
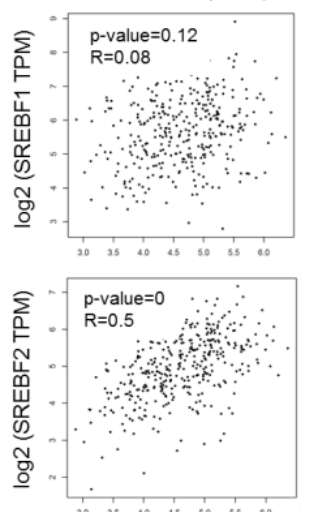

$\log 2(\mathrm{DAXX}$ TPM)
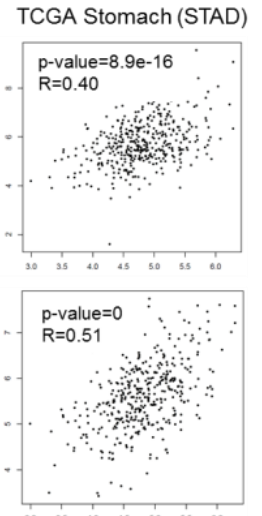

$\log 2$ (DAXX TPM)

TCGA Lymphoma (DLBC)
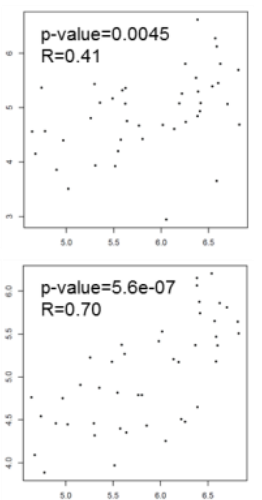

$\log 2$ (DAXX TPM)

TCGA Lung (LUAD)
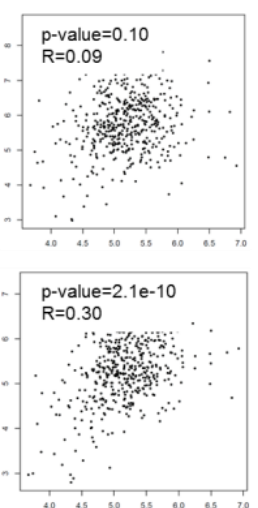

$\log 2$ (DAXX TPM)
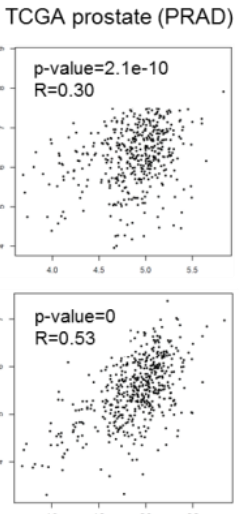

$\log 2$ (DAXX TPM)

TCGA pancreas (PAAD)

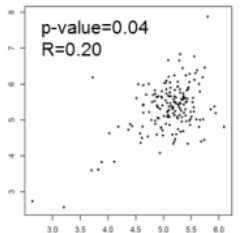

$p$-value $=0.00024$ ，

$R=0.30$

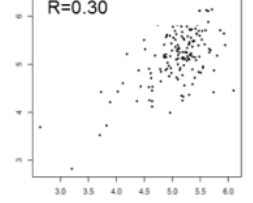

$\log 2$ (DAXX TPM)

TCGA Lung Squamous
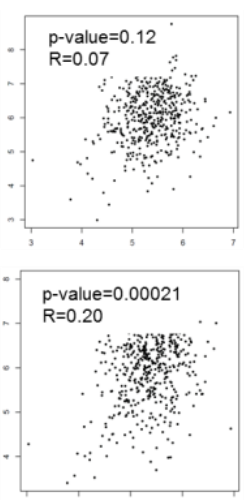

$\log 2$ (DAXX TPM)
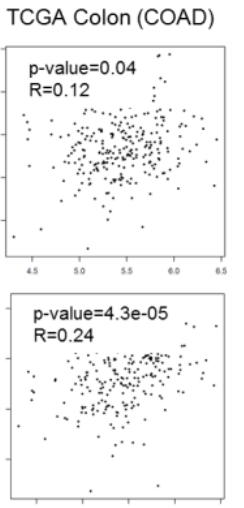

$\log 2$ (DAXX TPM)

TCGA Sarcoma (SARC)
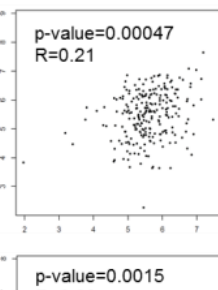

$\mathrm{R}=0.20$

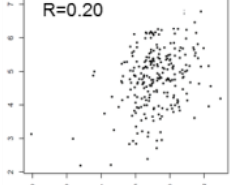

$\log 2$ (DAXX TPM)

TCGA Renal clear cell
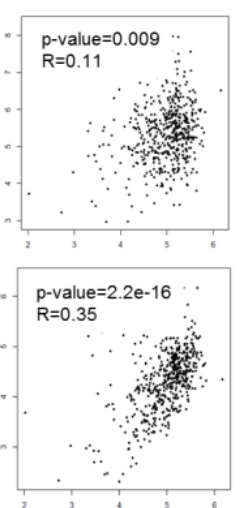

$\log 2$ (DAXX TPM)
TCGA Rectal (READ) TCGA Esophagous (ESCA)
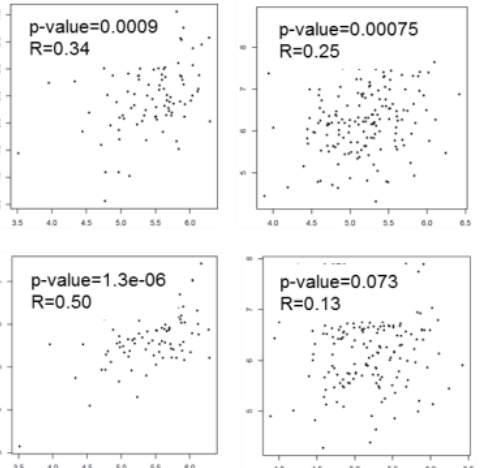

$\log 2($ DAXX TPM)

p-value $=0.073$

$\mathrm{R}=0.13$

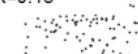

$\therefore \because$

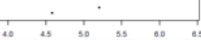

$\log 2$ (DAXX TPM)

TCGA Head (HNSC)

TCGA Ovary (OV)

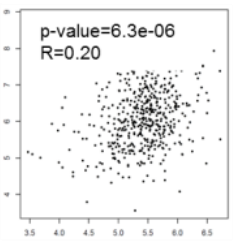

$\mathrm{p}$-value $=0.17$ $\mathrm{R}=0.06$

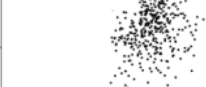

$\mathrm{p}$-value $=4.4 \mathrm{e}-11$

$\mathrm{R}=0.30$

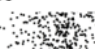

tis

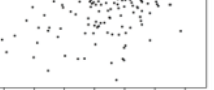

$\log 2$ (DAXX TPM)

TCGA Renal papillary
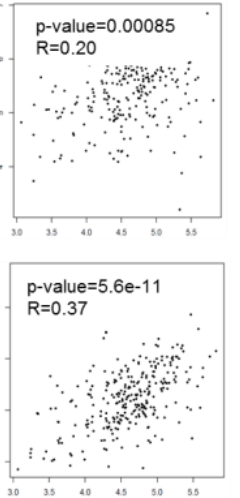

$\log 2$ (DAXX TPM)

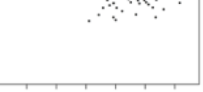

p-value $=3.8 \mathrm{e}-06$

$\mathrm{R}=0.20$

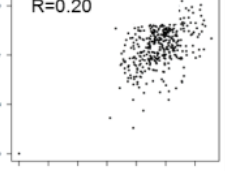

$\log 2$ (DAXX TPM)

TCGA Adrenal (ACC)

p-value $=0.32$

$\mathrm{R}=0.11$
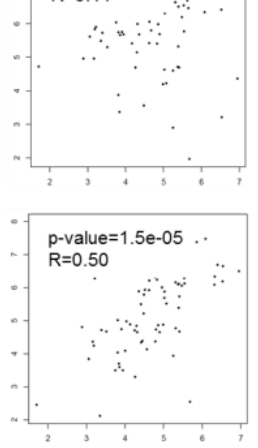

$\log 2$ (DAXX TPM)

Supplementary Figure S4. The mRNA expression of DAXX and SREBP1/2 is positively correlated in eighteen different human cancer types based on the Pearson correlation coefficient analysis of TCGA datasets. 
A

MDA-MB-231

\section{CTL DAXX}

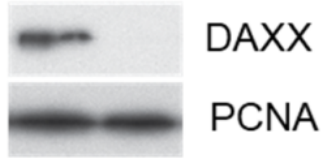

B

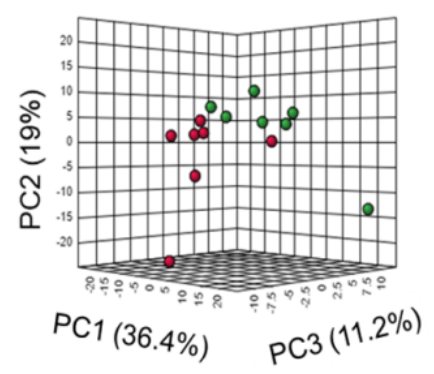

C

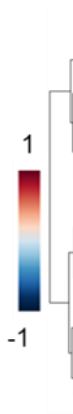

CTL_2

CTL_3

CTL_4

CTL_1

CTL 5

CRISTPR DAXX_6 CRISPR DAXX 3 CRISPR DAXX_4 CRISPR DAXX_1 CRISPR DAXX_2 CRISPR DAXX_5

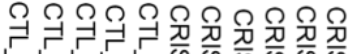
'N'W'

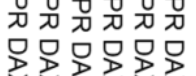

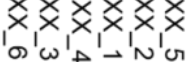

D

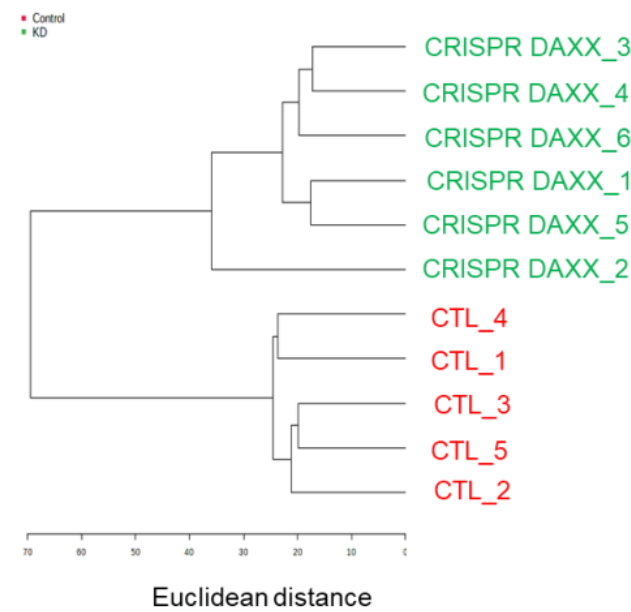

E

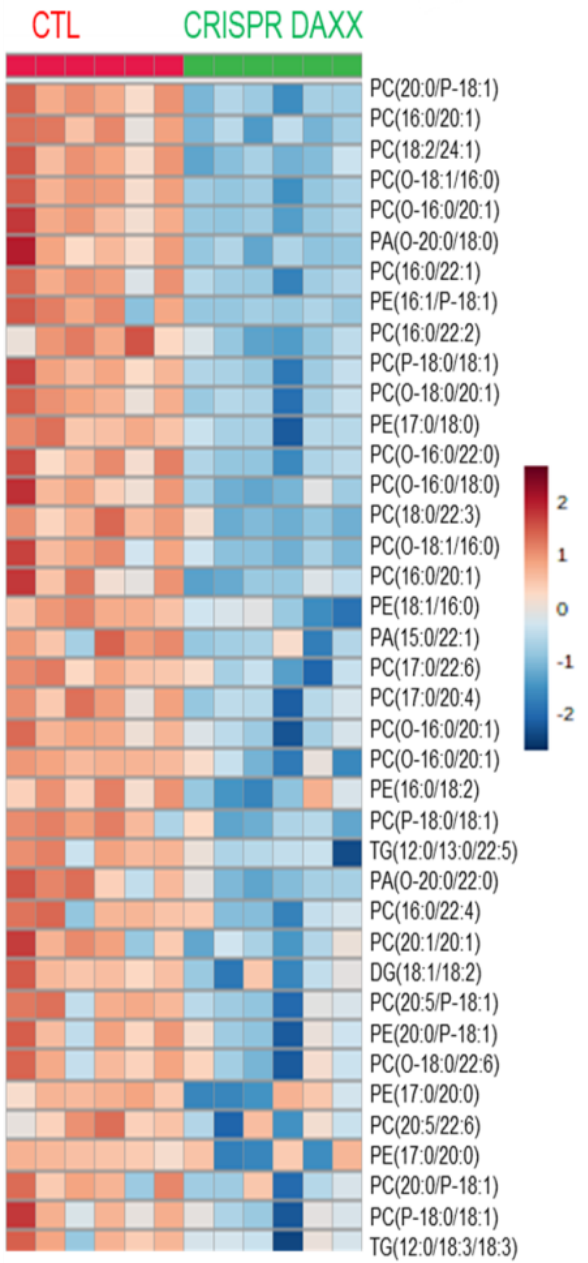

Supplementary Figure S5. CRISPR/Cas9-mediated DAXX depletion suppressed de novo lipogenesis.

(A) Western blot showing DAXX depletion in an MDA-MB-231 clone with a guide RNA targeting DAXX compared to control (CTL) cells.

(B) The PCA analysis of lipidomes in control (CTL, red dots) and CRISPR-DAXX MDA-MB-231 (green dots) cells $(n=6)$, which indicates distinct global lipid profiles in these two MDA-MB-231 cell lines.

(C) The Pearson correlation coefficient analysis of lipids in CTL and CRISPR-DAXX MDA-MB-231 cells show clear clustering of CTL and CRISPR-DAXX cells.

(D) The hierarchical clustering dendrogram analysis of lipids in CTL and CRISPR-DAXX MDA-MB-231 cells.

(E) The hierarchical heatmap analysis of lipids in CTL and CRISPR-DAXX MDA-MB-231 cells demonstrate reduced levels of specific lipid molecules in cells with DAXX depletion. 

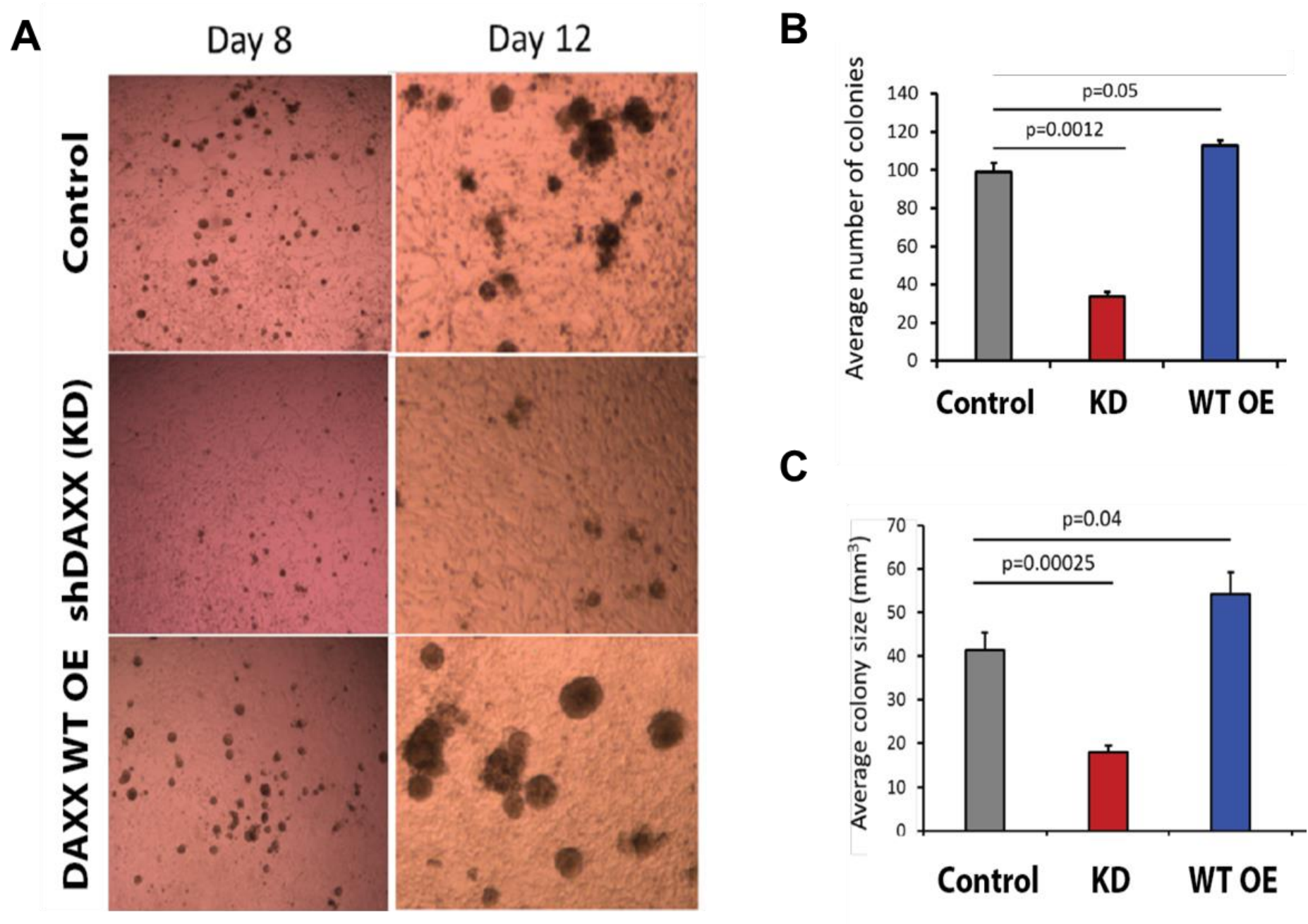

\section{Supplementary Figure S6. DAXX promotes cell proliferation and 3D colony growth in vitro.}

(A) MDA-MB-231-derived cell lines (Control, DAXX KD, and DAXX OE) were cultured in a suspension with Matrigel and complete DMEM medium. The 3D colonies of each line were imaged at the indicated time.

(B) Average colony number shown as bar graph and were quantified at Day 12.

(C) Average colony size shown as bar graph and were quantified at Day 12. 
A

\begin{tabular}{|c|c|c|c|}
\hline \multirow[b]{2}{*}{ de novo motif } & \multirow[b]{2}{*}{ Best match } & \multicolumn{2}{|r|}{ p value } \\
\hline & & Control & wT of \\
\hline 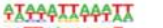 & $\mathrm{rno}$ & 10.50 & 2060 \\
\hline CTTCAGAGSGT & $\propto t$ & 10.28 & 1056 \\
\hline GTGGATCTACCA & zersisac & $10-07$ & sess \\
\hline CQSATGAAACG & was & 1est & 10,9 \\
\hline झСTCAACACCCA & $\operatorname{archa}$ & & $10-40$ \\
\hline GACTTGGT $\subset C C C$ & Net & 10313 & $10.2 \mathrm{~m}$ \\
\hline IITTTTATATAT & Net1 & . & $20-38$ \\
\hline ТТСТСАСАССТC & comen & & 1836 \\
\hline ACAGAAGICAAG & notos & & $10-35$ \\
\hline CGFCET ETAG & w & $\operatorname{ses} 4$ & se-3s \\
\hline GGGCCCAATGTA & moses. & & 10.33 \\
\hline TGTAGACC & zosiz & & sess \\
\hline$\triangle C A C$ CACX & zotos & & 10.33 \\
\hline \multirow[t]{2}{*}{ GATC $C$ GACC } & zases & & 10.23 \\
\hline & & & p value \\
\hline de novo motit & Best match & Control & WTOE \\
\hline CTGCACCAG & $\tan x(1-b a x)$ & $\cdot$ & $10-33$ \\
\hline SATK & Nex2-5 moneotiond & $\infty$ & $10-313$ \\
\hline TTCTGGGGTCT G & Runxa & $10-20$ & $10-32$ \\
\hline$A G C I C C A T G G$ & $\max$ & & 10.32 \\
\hline ATGTGSTISA & Runoce & $10-32$ & $20-32$ \\
\hline ACTCT C TSGG & $20+1$ & & 1022 \\
\hline AAT $T$ TGGGTC & $\cos 2$ & . & 1e 38 \\
\hline GCCTAACA & $\operatorname{sos}$ & & 10-19 \\
\hline CTCCAGACSC & smoses & & 2016 \\
\hline AACACCTG & roxas & set.7 & 1o-15 \\
\hline A. CACCTG & $\mathrm{Nu}(\mathrm{Cm})$ & sete & $10 \cdot 11$ \\
\hline ACACCTCT CT\& & 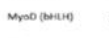 & 1007 & 10,11 \\
\hline \multirow[t]{2}{*}{ GCATAAAAS } & $\cos 2$ & se-30 & 2e-11 \\
\hline & & & $p$ value \\
\hline de novo motif & Best match & Control & WTOE \\
\hline CCSGICACGTGA & 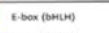 & . & $10-10$ \\
\hline$\triangle{ }^{C} \mathrm{CAC} T \mathrm{~T} G$ & omvetorasis & 1004 & 1e-11 \\
\hline AT CC소A & Sebpe (biven) & 1003 & 1aso \\
\hline$\therefore \mathrm{CAC} \mathrm{T} G \mathrm{~S} \mathrm{X}$ & Seboz (tarane & 10.02 & 18-11 \\
\hline AT GG TAGATCCA & nebs & 10.20 & 1063 \\
\hline \&STCACGTGA & usn & 10.04 & $10-10$ \\
\hline ATCACETGST & $u s \times 2$ & & $10-12$ \\
\hline AGGTCAOT TACCIS & $\ln (\operatorname{men})$ & 1e-04 & 1000 \\
\hline
\end{tabular}

B

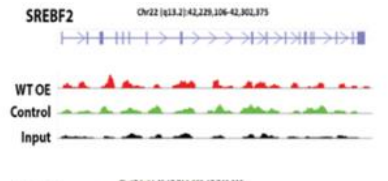

SREBF1 $\operatorname{sen}$ :

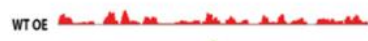
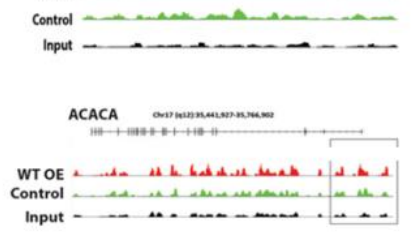

Input - _. . . . . . . . . .

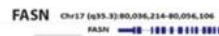

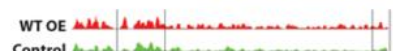

Control Let

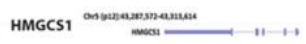

WT OE $1.1,1, \ldots+\ldots$
Control...

Control $\ldots \ldots \ldots$
Input $\perp \ldots$

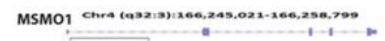

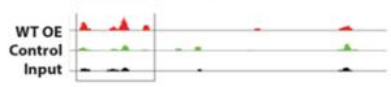

CYPS1A1

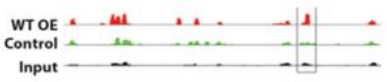

C

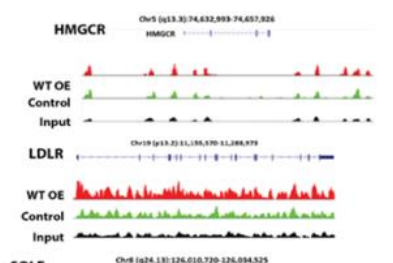

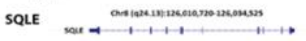

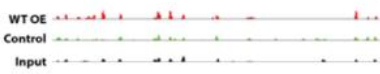

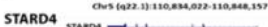

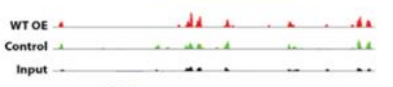

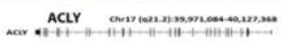

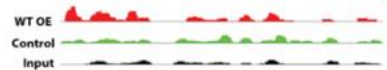

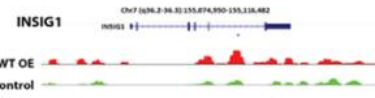

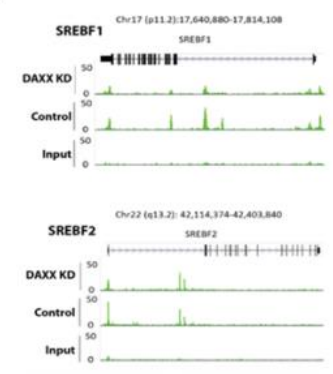

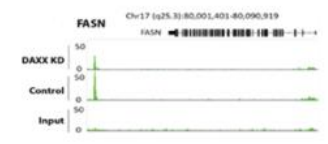

\section{Supplementary Figure S7. Chromatin-binding activity of DAXX.}

(A) De novo motifs associated with DAXX as revealed by ChIP-seq. DAXX ChIP-seq and motif analysis were done as in Figure 5. Motifs enriched in MDA-MB-231-derived cells (control and DAXX OE) are shown.

(B) DAXX chromatin-binding profiles of the indicated individual lipogenic genes in MDA-MB-231-derived cells (control and wt OE) are depicted.

(C) DAXX chromatin-binding profiles of the indicated individual lipogenic genes in PC3-derived cells (control vs. KD; Puto et al., 2015) are shown. 
A

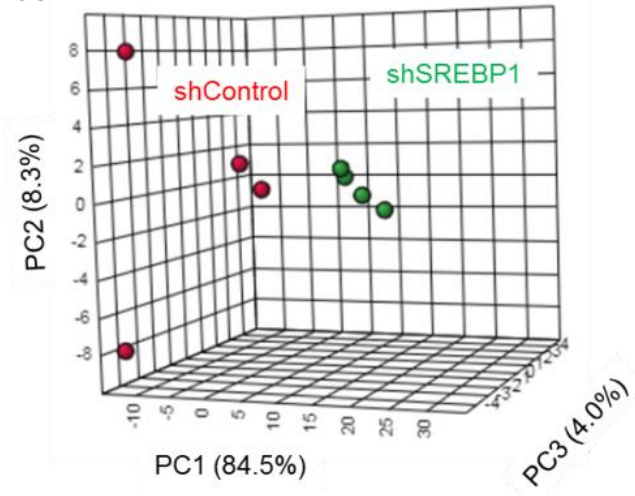

B

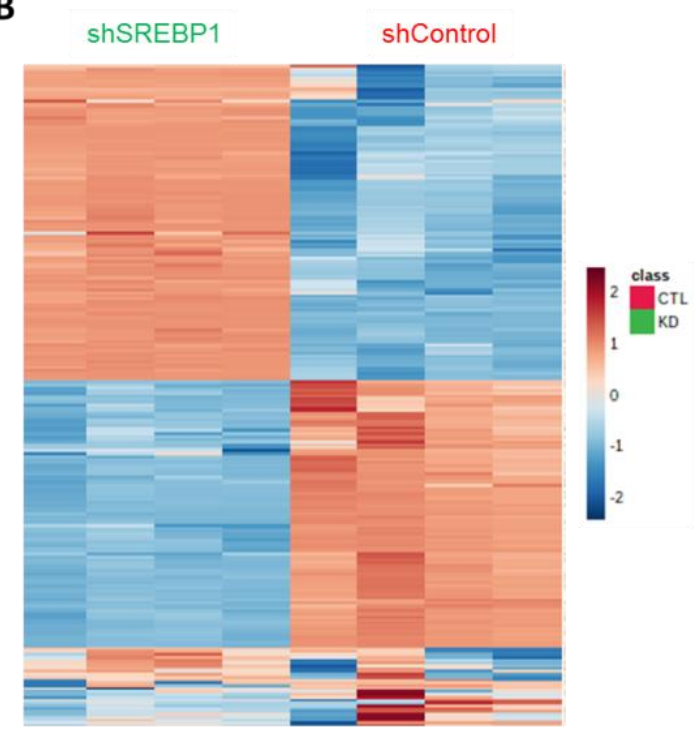

C

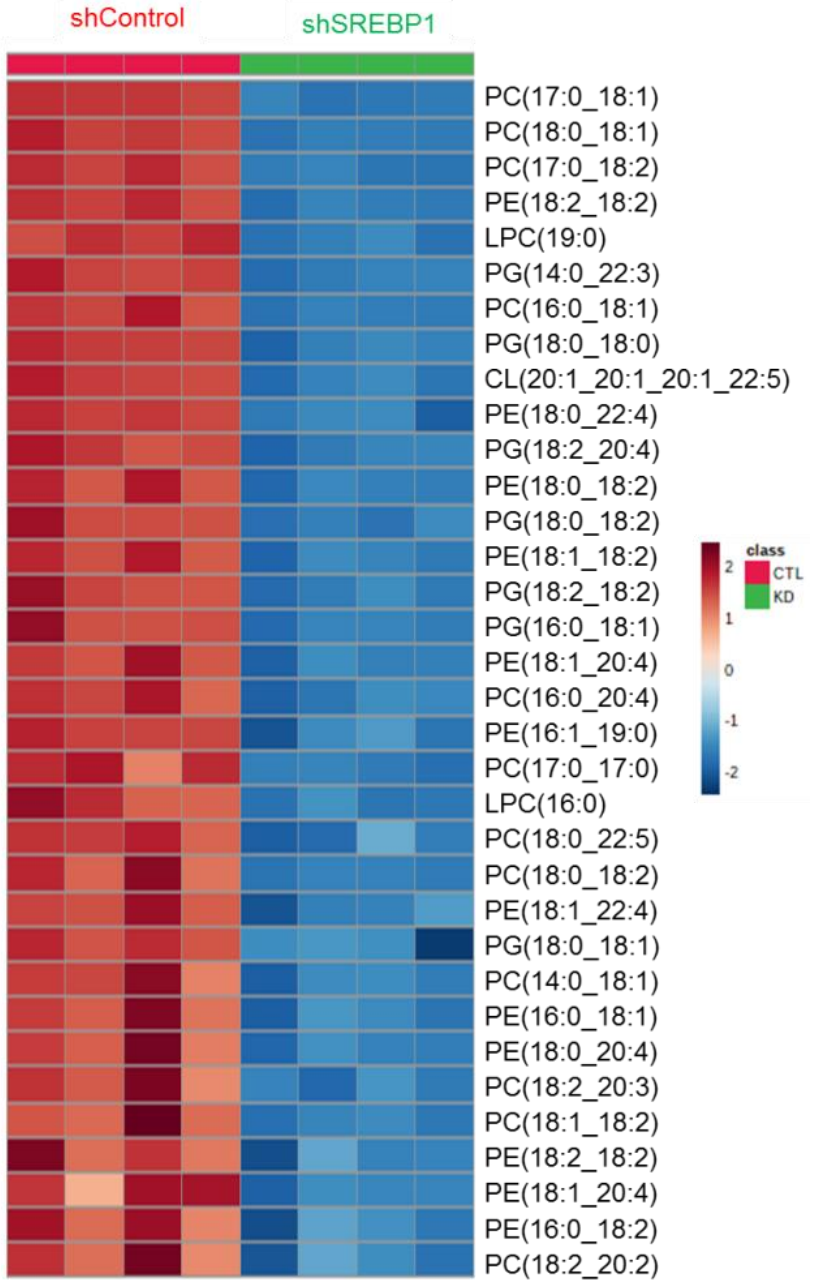

Supplementary Figure S8. Genetic knockdown of SREBP1 markedly impact global lipid profile.

(A) Principal component analysis comparing lipidomes between shControl and shSREBP1 cells derived from MDA-MB-231 cell line. Each dot represents an independent sample $(n=4)$.

(B) Hierarchical clustering heatmap analysis demonstrates global lipid landscape in shSREBP1 cells compared to shControl cells.

(C) Hierarchical clustering heatmap analysis of top differentially changed lipids including glycerolipid and glycerophospholipid molecules in MDA-MB-231 with SREBP1 KD compared to control cells. 


\section{Supplementary Table S1 PCR primers used for this study}

\begin{tabular}{|c|c|}
\hline \multicolumn{2}{|c|}{ RT-qPCR primers (5' to $3^{\prime}$ ) } \\
\hline ActinB-F-Real & GCTCCTCCTGAGCGCAAGTACTC \\
\hline ActinB-R-Real & GTGGACAGCGAGGCCAGGAT \\
\hline Daxx-RT-F & GAGGCGTCTCTCCTCACAAC \\
\hline Daxx-RT-R & TCTCATGCACTGACCTTTGC \\
\hline SREBP1-F & CTGCTGTCCACAAAAGCAAA \\
\hline SREBP1-R & GGTCAGTGTGTCCTCCACCT \\
\hline SREBP2-F & ATCGCTCCTCCATCAATGAC \\
\hline SREBP2-R & TTCCTCAGAACGCCAGACTT \\
\hline FASN-F & CACAGGGACAACCTGGAGTT \\
\hline FASN-R & ACTCCACAGGTGGGAACAAG \\
\hline HMGCR-F & GTCATTCCAGCCAAGGTTGT \\
\hline HMGCR-R & CATGGCAGAGCCCACTAAAT \\
\hline HMGCS1-F & GGGACACATATGCAACATGC \\
\hline HMGCS1-R & CACTGGGCATGGATCTTTTT \\
\hline FDPS-RT-F & CCAAGAAAAGCAGGATTTCG \\
\hline FDPS-RT-R & CCGGTTATACTTGCCTCCAA \\
\hline
\end{tabular}

\begin{tabular}{|l|l|}
\hline \multicolumn{2}{l}{ ChIP primers } \\
\hline FASN-prom-F1 & TAGAGGGAGCCAGAGAGACG \\
\hline FASN-prom-R1 & GCTGCTCGTACCTGGTGAG \\
\hline ACACA-prom-F1 & \\
\hline ACACA-prom-R1 & CAAGGGAAATTGAGGCTGAG \\
\hline SREBF-prom-F2 & \\
\hline SREBF-prom-R2 & TCCTTTAAACAAGGCGGAGA \\
\hline
\end{tabular}


bioRxiv preprint doi: https://doi.org/10.1101/2020.12.31.424997; this version posted January 3, 2021. The copyright holder for this preprint (which was not certified by peer review) is the author/funder. All rights reserved. No reuse allowed without permission.

\begin{tabular}{|l|l|}
\hline $\begin{array}{l}\text { SREBF2- } \\
\text { prom-F1 }\end{array}$ & CAGCTGAAGCTTGCATGCCTGCAGGTAGGCAGCTGGGAAGATGA \\
\hline $\begin{array}{l}\text { SREBF2- } \\
\text { prom-R1 }\end{array}$ & GAGTATATATAGGACTGGGGATCCGTGAGGGTCTCCATGGTCTC \\
\hline & $\begin{array}{l}\text { Note: The sequences in red are specific to the SREBF2 promoter region; and } \\
\text { the sequences in black correspond to the cloning vector }\end{array}$ \\
\hline
\end{tabular}




\section{Supplementary Table S2 Antibodies used in this study}

\begin{tabular}{|c|c|c|c|}
\hline Antibody target & Vendor/source & Vendor catalog \# & Dilution \\
\hline DAXX & Bethyl laboratories & A301-353A & $1: 20,000(\mathrm{IB})$ \\
\hline $\begin{array}{l}\text { DAXX (for IP, IB, IF } \\
\text { PLA, and ChIP) }\end{array}$ & $\begin{array}{l}\text { The Developmental Studies } \\
\text { Hybridoma Bank }\end{array}$ & PCRP-DAXX-5G11 & $\begin{array}{l}\text { Hybridoma } \\
\text { supernatant. 1:100 } \\
\text { for IB, 1:5 for IF, and } \\
1: 2 \text { for PLA }\end{array}$ \\
\hline DAXX (for IP and IB) & GenScript, rabbit polyclonal & This study & $1: 10,000$ for IB \\
\hline FASN (for IB and IF) & ProteinTech & 10624-2-AP & $\begin{array}{l}1: 20,000 \text { for IB, and } \\
1: 700 \text { for IF }\end{array}$ \\
\hline FASN & Santa Cruz & SC-55580 & $1: 20,000(\mathrm{IB})$ \\
\hline ACC1 & Cell Signaling Technology & 3676 & $1: 20,000$ (IB) \\
\hline ACsS2 & Cell Signaling Technology & 3658 & $1: 20,000(\mathrm{IB})$ \\
\hline SREBP2 & Abcam & ab30682 & $\begin{array}{l}1: 10,000 \text { for IB, } 1: 300 \\
\text { for IF and 1:100 for } \\
\text { PLA }\end{array}$ \\
\hline SREBP2 & BD Biosciences & 557037 & $1: 1,000(\mathrm{IB})$ \\
\hline SREBP1 & Santa Cruz & SC-13551 & $1: 5,000(\mathrm{IB})$ \\
\hline SREBP1 & ProteinTech & 4088-1-AP & $\begin{array}{l}1: 3,000 \text { for IB, } 1: 300 \\
\text { for IF and 1:100 for } \\
\text { PLA }\end{array}$ \\
\hline FLAG & Cell Signaling Technology & 14793 & $1: 10,000(\mathrm{IB})$ \\
\hline $\begin{array}{l}\text { FLAG (IB, IP and } \\
\text { ChIP) }\end{array}$ & Millipore-Sigma & F1804 & $1: 1,000(\mathrm{IB})$ \\
\hline GFP & Cell Signaling Technology & 2956 & $1: 3,000(\mathrm{IB})$ \\
\hline PCNA & Epitomics & $2714-1$ & $1: 20,000(\mathrm{IB})$ \\
\hline alpha-Tubulin & Millipore-Sigma & T5168 & $1: 50,000(\mathrm{IB})$ \\
\hline HSP60 & BD Transduction Laboratories & $\mathrm{H} 99020$ & $1: 50,000(\mathrm{IB})$ \\
\hline $\begin{array}{l}\text { Rabbit IgG HRP- } \\
\text { linked antibody }\end{array}$ & Cell Signaling Technology & 7074 & $1: 10,000(\mathrm{IB})$ \\
\hline $\begin{array}{l}\text { Mouse IgG HRP- } \\
\text { linked antibody }\end{array}$ & Cell Signaling Technology & 7076 & $1: 10,000(\mathrm{IB})$ \\
\hline $\begin{array}{l}\text { Normal mouse IgG } \\
\text { (for IP/ChIP control) }\end{array}$ & Santa Cruz & SC-2025 & \\
\hline
\end{tabular}

